\title{
Combined threshold and transverse momentum resummation for inclusive observables
}

\author{
Claudio Muselli, ${ }^{a}$ Stefano Forte $^{a}$ and Giovanni Ridolfi ${ }^{b}$ \\ ${ }^{a}$ Tif Lab, Dipartimento di Fisica, Università di Milano and INFN, Sezione di Milano, \\ Via Celoria 16, I-20133 Milano, Italy \\ ${ }^{b}$ Dipartimento di Fisica, Università di Genova and INFN, Sezione di Genova, \\ Via Dodecaneso 33, I-16146 Genova, Italy \\ E-mail: claudio.muselli@mi.infn.it, forte@mi.infn.it, \\ ridolfi@ge.infn.it
}

ABSTRACT: We present a combined resummation for the transverse momentum distribution of a colorless final state in perturbative QCD, expressed as a function of transverse momentum $p_{\mathrm{T}}$ and the scaling variable $x$. Its expression satisfies three requirements: it reduces to standard transverse momentum resummation to any desired logarithmic order in the limit $p_{\mathrm{T}} \rightarrow 0$ for fixed $x$, up to power suppressed corrections in $p_{\mathrm{T}}$; it reduces to threshold resummation to any desired logarithmic order in the limit $x \rightarrow 1$ for fixed $p_{\mathrm{T}}$, up to power suppressed correction in $1-x$; upon integration over transverse momentum it reproduces the resummation of the total cross cross at any given logarithmic order in the threshold $x \rightarrow 1$ limit, up to power suppressed correction in $1-x$. Its main ingredient, and our main new result, is a modified form of transverse momentum resummation, which leads to threshold resummation upon integration over $p_{T}$, and for which we provide a simple closed-form analytic expression in Fourier-Mellin $(b, N)$ space. We give explicit coefficients up to NNLL order for the specific case of Higgs production in gluon fusion in the effective field theory limit. Our result allows for a systematic improvement of the transverse momentum distribution through threshold resummation which holds for all $p_{\mathrm{T}}$, and elucidates the relation between transverse momentum resummation and threshold resummation at the inclusive level, specifically by providing within perturbative QCD a simple derivation of the main consequence of the so-called collinear anomaly of SCET.

KeYwords: Perturbative QCD, Resummation, Higgs Physics, Renormalization Group

ArXiv EPrint: 1701.01464 


\section{Contents}

1 Introduction 1

2 Factorization and resummation of transverse momentum distributions 2

2.1 Notations and kinematics 2

2.2 Factorization 4

2.3 Threshold resummation at large $p_{\mathrm{T}} \quad 5$

$\begin{array}{ll}2.4 & \text { Transverse momentum resummation }\end{array}$

3 Phase space factorization $\quad 8$

3.1 Large $N \quad 8$

$\begin{array}{llr}3.2 & \text { Small } p_{\mathrm{T}} & 12\end{array}$

4 Resummation $\quad 17$

$\begin{array}{ll}\text { 4.1 The combined resummed result } & 17\end{array}$

$\begin{array}{lll}4.2 & \text { Higgs production } & 21\end{array}$

5 Conclusions $\quad 28$

$\begin{array}{lr}\text { A Explicit expressions } & 29\end{array}$

$\begin{array}{lll}\text { A.1 Threshold resummation at fixed } p_{\mathrm{T}} & 29\end{array}$

A.2 Transverse momentum resummation 32

A.3 Threshold resummation for inclusive cross section 34

\section{Introduction}

Transverse momentum $p_{\mathrm{T}}$ distributions of inclusive observables, such as the Higgs or gaugeboson production cross sections, receive logarithmically enhanced corrections in perturbative $\mathrm{QCD}$, related to infrared and collinear radiation. While infrared radiation is also collinear, the opposite is not true. As a consequence, the all-order resummation of transverse momentum logarithms (transverse momentum resummation, henceforth) contains more information than the resummation of infrared threshold logarithms (threshold resummation, henceforth) of the total cross section, integrated over transverse momentum (total cross section, henceforth): it should therefore be possible to recover the latter from the former. On the other hand, the transverse momentum distribution in the threshold limit for finite $p_{\mathrm{T}}$ necessarily has a different logarithmic structure than the total cross section because there must be at least one non-soft emission recoiling against the final state. Therefore, the soft and collinear limits do not commute: if the collinear limit is taken first, extra logs are present which are not there when the soft limit is taken at finite $p_{\mathrm{T}}$, and conversely.

All-order techniques have been available since a long time for transverse momentum resummation [1], threshold resummation of total cross sections [2, 3], and more recently also for threshold resummation of transverse momentum distributions [4]. Their combination, 
however, is nontrivial because of the non-commutative nature of the soft and collinear limits. It is the purpose of this paper to provide such a combination. In particular, our goal is to construct an all-order resummed expression for transverse momentum distributions which has the following properties:

1. at small $p_{\mathrm{T}}$ it reproduces transverse momentum resummation to some fixed logarithmic accuracy;

2. in the threshold $x \rightarrow 1$ limit for finite $p_{\mathrm{T}}$ it reproduces threshold resummation also to some given accuracy;

3. upon integration over $p_{\mathrm{T}}$ it leads to a total cross section which reproduces threshold resummation to some logarithmic accuracy.

Some previous attempts to combined resummation have been presented, but they fail to simultaneously satisfy all of these criteria. Specifically, in ref. [5] a joint threshold and transverse momentum resummation has been presented which satisfies criteria 1 and 3 , but fails to satisfy criterion 2. This resummation, originally presented up to next-to-leading logarithmic (NLL) accuracy, was very recently extended to NNLL in the specific case of Drell-Yan production [6]. Also, the non-commutativity of the limits was recently recognized in refs. $[7,8]$, and the two resummations were combined at the differential level using SCET through a suitable tuning of the scales involved in such a way that the relevant limits do not mix. This leads to a result which satisfies criteria 1 and 2, but fails to satisfy criterion 3 : the integral over $p_{\mathrm{T}}$ does not reproduce the threshold resummation for inclusive cross section.

A crucial step towards the construction of our result is a careful analysis of the way phase space factorizes in the two limits we are interested in. Indeed, it turns out that in order to obtain either of the two resummations that we wish to combine, a different factorization of phase space is necessary, and also, that the threshold and small $p_{\mathrm{T}}$ limits do not commute because of the structure of phase space. This phase-space analysis will be presented in section 3, after an introductory section 2, in which various known individual resummed results are summarized and notation is introduced. Our combined resummed results will then be presented in section 4, with specific reference to the case of Higgs production, with conclusions drawn in section 5 .

\section{Factorization and resummation of transverse momentum distributions}

\subsection{Notations and kinematics}

We discuss the transverse momentum distribution of a colorless object which we shall refer to as "Higgs" for the sake of brevity, though it could just as well be e.g. a gauge boson. The factorized perturbative QCD expression for the Higgs $p_{\mathrm{T}}$ distribution at a hadron collider takes the form

$$
\begin{aligned}
\frac{d \sigma}{d \xi_{p}}\left(\tau, \xi_{p}, M^{2}\right)= & \sum_{i, j} \int_{\frac{\tau}{\left(\sqrt{1+\xi_{p}}-\sqrt{\xi_{p}}\right)^{2}}}^{1} d x_{1} \int_{\frac{\tau}{x_{1}\left(\sqrt{1+\xi_{p}}-\sqrt{\xi_{p}}\right)^{2}}}^{1} d x_{2} f_{i}\left(x_{1}, \mu_{\mathrm{F}}^{2}\right) f_{j}\left(x_{2}, \mu_{\mathrm{F}}^{2}\right) \\
& \frac{d \bar{\sigma}_{i j}}{d \xi_{p}}\left(\hat{\tau}, \xi_{p}, \alpha_{s}\left(\mu_{\mathrm{R}}^{2}\right), \mu_{\mathrm{F}}^{2}\right),
\end{aligned}
$$


where $\frac{d \sigma}{d \xi_{p}}$ and $\frac{d \bar{\sigma}_{i j}}{d \xi_{p}}$ are the hadronic and partonic distributions, written in terms of the dimensionless transverse momentum variable

$$
\xi_{p}=\frac{p_{\mathrm{T}}^{2}}{M^{2}}
$$

and the scaling variables

$$
\begin{aligned}
& \tau=\frac{M^{2}}{s} ; \\
& \hat{\tau}=\frac{M^{2}}{\hat{s}},
\end{aligned}
$$

where $M^{2}$ is the invariant Higgs mass, and $s$ and

$$
\hat{s}=x_{1} x_{2} s
$$

are respectively the hadronic and partonic center-of-mass squared energies. The partonic differential cross section is perturbatively determined in terms of the strong coupling $\alpha_{s}\left(\mu_{R}^{2}\right)$ at a renormalization scale $\mu_{R}$ and a factorization scale $\mu_{F}$, and $f_{i}\left(x_{1}, \mu_{\mathrm{F}}^{2}\right)$ are parton distributions.

Equation (2.1) can be rewritten as a convolution by defining

$$
\tau^{\prime}=\frac{Q^{2}}{s}
$$

where

Indeed, we then get

$$
Q^{2}=\left(\sqrt{M^{2}+p_{\mathrm{T}}^{2}}+p_{\mathrm{T}}\right)^{2}
$$

$$
\frac{d \sigma}{d \xi_{p}}\left(\tau, \xi_{p}, M^{2}\right)=\tau^{\prime} \sum_{i j} \int_{\tau^{\prime}}^{1} \frac{d x}{x} \mathcal{L}_{i j}\left(\frac{\tau^{\prime}}{x}, \mu_{\mathrm{F}}^{2}\right) \frac{1}{x} \frac{d \hat{\sigma}_{i j}}{d \xi_{p}}\left(x, \xi_{p}, \alpha_{s}\left(\mu_{\mathrm{R}}^{2}\right), \mu_{\mathrm{F}}^{2}\right),
$$

where the parton luminosity is defined in the usual way as

$$
\mathcal{L}_{i j}\left(x, \mu_{\mathrm{F}}^{2}\right)=\int_{x}^{1} \frac{d y}{y} f_{i}\left(y, \mu_{\mathrm{F}}^{2}\right) f_{j}\left(\frac{x}{y}, \mu_{\mathrm{F}}^{2}\right),
$$

and

$$
\frac{d \hat{\sigma}_{i j}}{d \xi_{p}}\left(x, \xi_{p}, \alpha_{s}\left(\mu_{\mathrm{R}}^{2}\right), \mu_{\mathrm{F}}^{2}\right)=\frac{d \bar{\sigma}_{i j}}{d \xi_{p}}\left(\frac{x}{\left(\sqrt{1+\xi_{p}}+\sqrt{\xi_{p}}\right)^{2}}, \xi_{p}, \alpha_{s}\left(\mu_{\mathrm{R}}^{2}\right), \mu_{\mathrm{F}}^{2}\right),
$$

so that the partonic scaling variable in eq. (2.8) is

$$
x=\frac{Q^{2}}{\hat{s}}=\frac{M^{2}}{\hat{s}}\left(\sqrt{1+\xi_{p}}+\sqrt{\xi_{p}}\right)^{2} .
$$

The scale variable $Q^{2}$ eq. (2.7) has a straightforward physical interpretation, as the minimum allowed value of the invariant mass of the final state at fixed transverse momentum. Consequently, the threshold limit corresponds to the limit $\tau^{\prime} \rightarrow 1$ of the scaling variable eq. (2.6), and at the partonic level it corresponds to the integration region $x \sim 1$ in eq. (2.8). 


\subsection{Factorization}

Threshold resummation is most naturally performed on the Mellin transform of the cross section. Performing a Mellin transform with respect to $\tau^{\prime}$ the convolution eq. (2.8) factorizes. Indeed, defining

$$
\begin{aligned}
\frac{d \sigma}{d \xi_{p}}\left(N, \xi_{p}\right) & =\int_{0}^{1} d \tau^{\prime} \tau^{\prime N-1} \frac{d \sigma}{d \xi_{p}}\left(\tau, \xi_{p}\right) ; \\
\frac{d \hat{\sigma}_{i j}}{d \xi_{p}}\left(N, \xi_{p}\right) & =\int_{0}^{1} d x x^{N-1} \frac{d \hat{\sigma}_{i j}}{d \xi_{p}}\left(x, \xi_{p}\right),
\end{aligned}
$$

where with a slight abuse of notation we are using the same symbol for the cross section and its Mellin transform, we get

$$
\frac{d \sigma}{d \xi_{p}}\left(N, \xi_{p}, M^{2}\right)=\sum_{i j} \mathcal{L}_{i j}\left(N+1, \mu_{\mathrm{F}}^{2}\right) \frac{d \hat{\sigma}_{i j}}{d \xi_{p}}\left(N, \xi_{p}, \alpha_{s}\left(\mu_{\mathrm{R}}^{2}\right), \mu_{\mathrm{F}}^{2}\right) .
$$

It is important to observe that, because the scale $Q^{2}$ eq. (2.7) and consequently the scaling variable $\tau^{\prime}$ eq. (2.6) both depend on $p_{\mathrm{T}}$, the Mellin transforms of the total hadronic and partonic cross sections

$$
\begin{aligned}
\sigma(\tau) & =\int_{0}^{\frac{(1-\tau)^{2}}{4 \tau}} d \xi \frac{d \sigma}{d \xi}(\tau, \xi) \\
\hat{\sigma}_{i j}(\hat{\tau}) & =\int_{0}^{\frac{(1-\hat{\tau})^{2}}{4 \hat{\tau}}} d \xi \frac{d \bar{\sigma}_{i j}}{d \xi}(\hat{\tau}, \xi)
\end{aligned}
$$

are not just the integrals over $p_{\mathrm{T}}$ of the Mellin transforms eq. (2.12), (2.13) of the respective transverse momentum distributions. Rather, defining the Mellin transforms of the total hadronic and partonic cross sections

$$
\begin{aligned}
\sigma(N) & =\int_{0}^{1} d \tau \tau^{N-1} \sigma(\tau) \\
\hat{\sigma}_{i j}(N) & =\int_{0}^{1} d \hat{\tau} \hat{\tau}^{N-1} \hat{\sigma}_{i j}(\hat{\tau})
\end{aligned}
$$

which factorize the cross section as

$$
\sigma\left(N, \alpha_{s}\left(\mu_{\mathrm{R}}^{2}\right), \mu_{\mathrm{F}}^{2}\right)=\sum_{i j} \mathcal{L}_{i j}\left(N+1, \mu_{\mathrm{F}}^{2}\right) \hat{\sigma}_{i j}\left(N, M^{2}\right),
$$

and using eqs. (2.11), (2.16) we get

$$
\hat{\sigma}_{i j}(N)=\int_{0}^{\infty} d \xi_{p}\left(\sqrt{1+\xi_{p}}-\sqrt{\xi_{p}}\right)^{2 N} \frac{d \hat{\sigma}_{i j}}{d \xi_{p}}\left(N, \xi_{p}\right) .
$$

It is very important to observe that here and everywhere henceforth we will assume that the Mellin transform is defined for transverse momentum distributions according to eqs. (2.12)(2.13) (with the partonic differential cross section $\frac{d \hat{\sigma}_{i j}}{d \xi_{p}}\left(N, \xi_{p}\right)$ given by eq. (2.10)), and for 
total cross sections according to eqs. (2.17)-(2.18). They are thus related by eq. (2.20), as a consequence of the fact that the integral transformation kernel differs between the two cases.

Transverse momentum resummation is best performed on the Fourier transform of the transverse momentum distribution. This is defined as

$$
\frac{d \hat{\sigma}_{i j}}{d \xi_{p}}(N, b)=\frac{1}{M^{2}} \int d^{2} p_{\mathrm{T}} e^{i \vec{b} \cdot \vec{p}_{\mathrm{T}}} \frac{d \hat{\sigma}_{i j}}{d \xi_{p}}\left(N, \xi_{p}\right),
$$

which, performing the angular integration, becomes

$$
\frac{d \hat{\sigma}_{i j}}{d \xi_{p}}(N, b)=\pi \int_{0}^{\infty} d \xi_{p} J_{0}\left(b p_{\mathrm{T}}\right) \frac{d \hat{\sigma}_{i j}}{d \xi_{p}}\left(N, \xi_{p}\right),
$$

where $J_{0}$ is the Bessel function.

It is interesting to observe that we are free to take a simultaneous Mellin and Fourier transform thanks to the fact that the Mellin transform is performed, according to eq. (2.12), with respect to the scaling variable $\tau^{\prime}$ eq. (2.6). Indeed, the variable $\tau^{\prime}$ ranges from $0 \leq \tau^{\prime} \leq 1$ for all $p_{\mathrm{T}}$, and $p_{\mathrm{T}}$ ranges from $0 \leq p_{\mathrm{T}} \leq \infty$ for all $\tau^{\prime}$. This is to be contrasted to the the situation in which the kinematics is parametrized by $p_{\mathrm{T}}$ and the scaling variable $\tau$ eq. (2.3). In that case, for fixed $p_{\mathrm{T}}, \tau$ has a $p_{\mathrm{T}}$-dependent upper boundary:

$$
0 \leq \tau \leq\left(\sqrt{1+\xi_{p}}-\sqrt{\xi_{p}}\right)^{2} .
$$

Conversely, for fixed $\tau, p_{\mathrm{T}}$ has a $\tau$-dependent range, most easily expressed in terms of the dimensionless variable $\xi_{p}$ :

$$
0 \leq \xi_{p} \leq \frac{(1-\tau)^{2}}{4 \tau} .
$$

Hence, it is not possible to take a Mellin transform with respect to $\tau$ of the $p_{\mathrm{T}}$ distribution, or a Fourier transform with respect to $p_{\mathrm{T}}$ at fixed $\tau$, without extending the integration range outside the physical region. Note, however, that when $p_{\mathrm{T}} \rightarrow 0$ the upper limit of $\tau$ eq. (2.23) tends to one. Equivalently,

$$
\tau^{\prime}=\tau\left(1+O\left(\sqrt{\xi}_{p}\right)\right)
$$

It follows that transverse momentum resummation can be performed using the scaling variable $\tau$ eq. (2.3) up to corrections which are power-suppressed in the small- $p_{\mathrm{T}}$ limit. However, in order to obtain results which hold also when $p_{\mathrm{T}}$ is kept finite, as it is our goal, the scaling variable $\tau^{\prime}$ eq. (2.6) must be used.

\subsection{Threshold resummation at large $p_{\mathrm{T}}$}

Threshold resummation for the $p_{\mathrm{T}}$ distribution of colorless final states was presented in ref. [4]. It is based on the observation that it is merely a particular case of soft resummation of a process which at leading-order has more than one parton in the final state [9], such as prompt-photon production [10], which corresponds to the particular case in which the mass $M$ which enters the definition of the scale variable eq. (2.7) vanishes. 
As discussed in refs. $[10,11]$, soft resummation in this case is characterized by the fact that there are two different classes of soft emissions, characterized by two different scales: one related to the emission of soft partons (akin to that for soft resummation in gauge or Higgs boson production) and one related to emission of partons which are collinear to the parton whose transverse momentum balances that of the colorless final state. Soft partons, in turn, can be emitted either collinear to the incoming leg, or at large angle: the contribution from the latter then depends on $p_{\mathrm{T}}$, as we shall see more explicitly in section 3.1 below.

Threshold resummation of transverse momentum distributions takes the form

$$
\frac{d \hat{\sigma}_{i j}^{\text {th }}}{d \xi_{p}}\left(N, \xi_{p}, \alpha_{s}\left(Q^{2}\right), Q^{2}\right)=\sigma_{0} C_{0}\left(N, \xi_{p}\right) g_{0 i j}\left(\xi_{p}\right) \exp [G(N)] \exp \left[S\left(N, p_{\mathrm{T}}\right)\right],
$$

where $Q^{2}$ is the scale eq. (2.7), and the resummation of soft large-angle emissions is included in the $p_{\mathrm{T}}$-dependent soft function $S\left(N, p_{\mathrm{T}}\right)$, while all other soft and collinear emissions are resummed into the $p_{\mathrm{T}}$-independent Sudakov exponent $G(N)$. All contributions which do not vanish as $N \rightarrow \infty$ but are also not logarithmically enhanced are contained in the matching function $\sigma_{0} C_{0}\left(N, \xi_{p}\right) g_{0} i j\left(\xi_{p}\right)$ which is a power series in $\alpha_{s}$, with $\sigma_{0}$ the leading-order $(\mathrm{LO})$ inclusive cross section and $\sigma_{0} C_{0}\left(N, \xi_{p}\right)$ the unresummed LO transverse momentum distribution.

The Sudakov exponent has the structure

$$
G(N)=\Delta_{i}(N)+\Delta_{j}(N)+J_{k}(N),
$$

where $i, j$ are the initial partons, $k$ is the final hard recoiling parton, and $i, j, k=g, q$. Also,

$$
\begin{aligned}
& \Delta_{i}(N)=\int_{0}^{1} d z \frac{z^{N-1}-1}{1-z} \int_{Q^{2}}^{Q^{2}(1-z)^{2}} \frac{d q^{2}}{q^{2}} A_{i}^{\text {th }}\left(\alpha_{s}\left(q^{2}\right)\right) \\
& J_{k}(N)=\int_{0}^{1} d z \frac{z^{N-1}-1}{1-z} \int_{Q^{2}(1-z)^{2}}^{Q^{2}(1-z)} \frac{d q^{2}}{q^{2}} A_{k}^{\text {th }}\left(\alpha_{s}\left(q^{2}\right)\right)+B_{k}^{\text {th }}\left(\alpha_{s}\left(Q^{2}(1-z)\right)\right),
\end{aligned}
$$

where $A_{i}^{\text {th }}\left(\alpha_{s}\right)$ and $B_{i}^{\text {th }}\left(\alpha_{s}\right)$ are power series in $\alpha_{s}$ with numerical coefficients.

The soft exponent is entirely determined by the soft anomalous dimension, and thus depends only on the partons which enter the LO process: at any order in $\alpha_{s}$ it takes the form $[12,13]$

$$
S\left(N, \xi_{p}\right)=-\int_{0}^{1} d z \frac{z^{N-1}-1}{1-z} A_{k}^{\text {th }}\left(\alpha_{s}\left(Q^{2}(1-z)^{2}\right)\right) \ln \frac{\left(\sqrt{1+\xi_{p}}+\sqrt{\xi_{p}}\right)^{2}}{\xi_{p}},
$$

where $A_{k}^{\text {th }}$ is the so-called cusp anomalous dimension, i.e. the coefficient of the most singular contribution to the anomalous dimension as $x \rightarrow 1$ and $k=g, q$ the type of the recoiling hard final parton. ${ }^{1}$ It follows that $G(N)$ and $S\left(N, \xi_{p}\right)$ eqs. (2.27)-(2.30) can be entirely

\footnotetext{
${ }^{1}$ When comparing the form given in eqs. (2.26)-(2.30) of the resummation to that given in eqs. (12)-(17) of ref. [4] it should be kept in mind that in that reference the dependence on $p_{\mathrm{T}}$ is given through a variable $r$ defined as $r=\sqrt{\frac{\xi_{p}}{1+\xi_{p}}}$. Also, the scale variable $Q^{2}$ in that reference is chosen as $Q^{2}=p_{\mathrm{T}}^{2} \frac{1+r}{r}$, which differs from our choice eq. (2.7). This difference can be reabsorbed by suitably redefining order by order the coefficients of the expansion of the functions $A$ and $B$ and the expression of $S$.
} 
derived from knowledge of threshold resummation at the inclusive level; coefficients up to NNLL are given in ref. [14]. The matching function can be determined up to $\mathrm{N}^{k}$ LL by matching to the fixed $\mathrm{N}^{k} \mathrm{LO}$ result.

It is important to observe that the leading-order cross section $\frac{d \hat{\sigma}_{j}^{\mathrm{LO}}}{d \xi_{p}}\left(N, \xi_{p}\right)$ vanishes as $\frac{1}{\sqrt{N}}$ as $N \rightarrow \infty$; correspondingly, the $\tau$-space partonic cross section is an ordinary function, rather than a distribution, unlike the partonic total Drell-Yan production cross section, and like the heavy quark production cross section, as discussed in ref. [15].

\subsection{Transverse momentum resummation}

Transverse momentum resummation was first formalized in ref. [1]. Here we use the form of it given in refs. [16, 17]: the partonic resummed cross section has the form

$$
\frac{d \hat{\sigma}_{i j}^{\mathrm{tr}}}{d \xi_{p}}\left(N, \xi_{p}, \alpha_{s}\left(M^{2}\right), M^{2}\right)=\sigma_{0} \int_{0}^{\infty} d b \frac{b}{2} J_{0}\left(b p_{\mathrm{T}}\right) H_{i j}\left(N, \alpha_{s}\left(M^{2}\right)\right) S(M, b)+\mathcal{O}\left(\frac{1}{b}\right),
$$

where we have chosen $\mu_{R}^{2}=\mu_{F}^{2}=M^{2}, \sigma_{0}$ is the LO inclusive cross section, the hard cross section $H_{i j}\left(N, \alpha_{s}\left(M^{2}\right)\right)$ is evaluated at fixed order in perturbation theory, and the Sudakov form factor $S(M, b)$ is given by

$$
S(M, b)=\exp \left[-\int_{\frac{b_{0}^{2}}{b^{2}}}^{M^{2}} \frac{d q^{2}}{q^{2}}\left[A^{p_{\mathrm{T}}}\left(\alpha_{s}\left(q^{2}\right)\right) \ln \frac{M^{2}}{q^{2}}+B^{p_{\mathrm{T}}}\left(\alpha_{s}\left(q^{2}\right), N\right)\right]\right]+\mathcal{O}\left(\frac{1}{b}\right) .
$$

Both $A^{p_{\mathrm{T}}}$ and $B^{p_{\mathrm{T}}}$ and the hard function are series in $\alpha_{s} ; A^{p_{\mathrm{T}}}$ has purely numerical coefficients while all the $N$-dependence at the exponent is contained in $B^{p_{T}}$ which starts at NLL level. Moreover, we factorize the LO inclusive cross section in eq. (2.31) so that $H_{i j}=1+\mathcal{O}\left(\alpha_{s}\right)$. The leading logarithmic contribution is fully determined by leading-order $A^{p_{\mathrm{T}}}$, and each contribution to $A^{p_{\mathrm{T}}}$ is enhanced by one logarithmic order in comparison to the contribution to $B^{p_{\mathrm{T}}}$ of the same order in $\alpha_{s}$ because of the explicit logarithm multiplying it in eq. (2.32). An $N$-independent contribution to $B^{p_{\mathrm{T}}}$ can be traded for a contribution to $A^{p_{\mathrm{T}}}$ with one less power of $\alpha_{s}$, however, because $A^{p_{\mathrm{T}}}$ starts to contribute at LL and $B^{p_{\mathrm{T}}}$ at NLL, the separation into $A^{p_{\mathrm{T}}}$ and $B^{p_{\mathrm{T}}}$ is uniquely determined, e.g. by expanding out eq. (2.31) and matching to the fixed-order computation. The physical meaning of $A^{p_{\mathrm{T}}}$ and $B^{p_{\mathrm{T}}}$ will become clear once we work out the relation between transverse momentum resummation and soft resummation at the inclusive level, and will be discussed in the end of section 4. As a byproduct, we will derive the relation between $A^{p_{\mathrm{T}}}$ and the cusp anomalous dimension $A^{\text {th }}$. The fact that these two quantities differ was originally found in ref. [18], where it was shown to follow in a SCET approach from a peculiar property of the effective Lagrangian dubbed "collinear anomaly": our result will provide a complementary derivation using standard perturbative QCD techniques.

Note that in ref. [1] the scale $\mu_{F}$ at which the parton distributions are evaluated is chosen to be a soft scale $\mu_{F}=\frac{1}{b}$. The difference can be reabsorbed into the function $B^{p_{\mathrm{T}}}$; the choice made here leads to a simpler relation between transverse momentum resummation and soft resummation of the inclusive cross section. Because it contains the evolution of the PDFs from the scale $\frac{1}{b}$ to the scale $M^{2}, B^{p_{\mathrm{T}}}$ is a matrix in flavor space, determined 
in terms of the anomalous dimension matrix. The exponential in eq. (2.31) should thus be interpreted as path-ordered. Note finally that there is a certain latitude in defining the function $H_{i j}\left(N, \alpha_{s}\left(M^{2}\right)\right)$, which includes terms which are not logarithmically enhanced as $p_{\mathrm{T}} \rightarrow 0$ and is computed to finite order in perturbation theory by matching to the finite order result. This freedom modifies the Sudakov form factor from NNLL onward, because a constant (i.e. non-logarithmic) term is of the same logarithmic order as a NNLL contribution. It can thus be viewed as a resummation scheme ambiguity. In the sequel we will adopt the hard resummation scheme defined in ref. [19].

\section{Phase space factorization}

It was shown in ref. [20] (and more recently using effective field theory arguments, see ref. [21]) that transverse momentum and threshold resummation can both be derived by using the renormalization group from a suitable factorization of the cross section. In ref. [22] this factorization was proven for inclusive colorless observables in the threshold limit; this argument was generalized in ref. [11] to the case of prompt-photon production, whose resummation, as mentioned in section 2.3 above, can be viewed as a particular case of threshold resummation at fixed $p_{\mathrm{T}}$.

The factorization argument of refs. $[11,22]$ relies on a kinematical analysis of phasespace for the process

$$
g\left(p_{1}\right)+g\left(p_{2}\right) \rightarrow \mathcal{S}(p)+g\left(k_{1}\right)+\cdots+g\left(k_{n}\right),
$$

where $\mathcal{S}$ is a colorless system and $g$ are massless gluons. We will now review this argument and show how it relates to the resummed result eq. (2.26). This analysis will elucidate why threshold resummation at fixed $p_{\mathrm{T}}$ only resums a subset of the soft radiation which is included in transverse momentum resummation. We will then discuss the different factorization of phase space which is needed in order to perform transverse momentum resummation. It will then be clear how to modify this latter factorization by the inclusion of suitable subleading terms such that the threshold limit is not spoiled, and in particular threshold resummation at the inclusive level follows from it, though still not the full threshold resummation at fixed $p_{\mathrm{T}}$. The simultaneous transverse momentum and threshold resummation for all $p_{\mathrm{T}}$ will involve matching these two different forms of factorization, and will be presented in the next section.

\subsection{Large $N$}

The phase-space factorization of refs. $[11,22]$ is based on the iterative reduction of the $n+1$ body phase space $d \Phi$ for the process eq. (3.1) into an $n$-body and a two-body phase space, which eventually leads to expressing it in terms of $n$ two-body phase spaces, connected by integrations over intermediate virtual particle masses. Physically, this corresponds to iteratively writing the phase space in terms of the momentum of the last radiated particle, 
and the system containing the previous $n-1$ final-state ones:

$$
\begin{aligned}
d \Phi\left(p_{1}, p_{2} ; k_{1}, \ldots, k_{n}, p\right)= & \frac{d P_{n}^{2}}{2 \pi} d \Phi\left(p_{1}, p_{2} ; k_{n}, P_{n}\right) \frac{d P_{n-1}^{2}}{2 \pi} d \Phi\left(P_{n} ; k_{n-1}, P_{n-1}\right) \\
& \ldots \frac{d P_{2}^{2}}{2 \pi} d \Phi\left(P_{3} ; k_{2}, P_{2}\right) d \Phi\left(P_{2} ; k_{1}, p\right),
\end{aligned}
$$

where each of the intermediate particle's invariant mass $P_{i}^{2}$ ranges between

$$
M^{2} \leq P_{i}^{2} \leq P_{i+1}^{2}
$$

and $P_{n+1}^{2} \equiv \hat{s}$ is the total center-of-mass energy squared.

The result is then simplified taking advantage of the Lorentz invariance of each twobody phase space, in order to rewrite it in the rest frame of its incoming momentum $P_{i}$ : in $d=4-2 \epsilon$ dimensions

$$
\begin{aligned}
d \Phi\left(P_{i} ; k_{j}, P_{j}\right) & =\frac{(2 \pi)^{2-d}}{4} \frac{d^{d-1} k_{j}}{\left|\vec{k}_{j}\right| \sqrt{\left|\vec{k}_{j}\right|^{2}+P_{j}^{2}}} \delta\left(\sqrt{P_{i}^{2}}-\sqrt{\left|\vec{k}_{j}\right|^{2}+P_{j}^{2}}-\left|\vec{k}_{j}\right|\right) \\
& =\frac{(4 \pi)^{2 \epsilon-2}}{2}\left(P_{i}^{2}\right)^{-\epsilon}\left(1-\frac{P_{j}^{2}}{P_{i}^{2}}\right)^{1-2 \epsilon}\left(\sin \theta_{j}\right)^{1-2 \epsilon} d \theta_{j} d \Omega_{2-2 \epsilon}^{j} \\
& =\frac{(2 \pi)^{2 \epsilon-2}}{8}\left(k_{\mathrm{T}_{\mathrm{j}}}^{2}\right)^{-\epsilon} \frac{d k_{\mathrm{T}_{\mathrm{j}}}^{2} d \Omega_{2-2 \epsilon}^{j}}{\sqrt{P_{i}^{2}} \sqrt{\frac{P_{i}^{2}}{4}\left(1-\frac{P_{j}^{2}}{P_{i}^{2}}\right)^{2}-k_{\mathrm{T}_{\mathrm{j}}}^{2}}},
\end{aligned}
$$

where the angular integral is written in terms of a $(1-2 \epsilon)$-dimensional azimuthal integration over $d \Omega_{2-2 \epsilon}^{i}$, and, equivalently, either a polar integral over $\theta_{i}$ in eq. (3.4), or the modulus square of the transverse momentum in eq. (3.5). In the latter case, the squareroot factor in the denominator of eq. (3.5) is the Jacobian related to this new choice of integration variable.

Using this result the $d$-dimensional phase space eq. (3.2) becomes

$$
\begin{aligned}
d \Phi= & (4 \pi)^{2 \epsilon-3}\left(P_{n+1}^{2}\right)^{-\epsilon}\left(1-\frac{P_{n}^{2}}{P_{n+1}^{2}}\right)^{1-2 \epsilon}\left(\sin \theta_{n}\right)^{1-2 \epsilon} d \theta_{n} d \Omega_{2-2 \epsilon}^{n} d P_{n}^{2} \\
& \times(4 \pi)^{2 \epsilon-3}\left(P_{n}^{2}\right)^{-\epsilon}\left(1-\frac{P_{n-1}^{2}}{P_{n}^{2}}\right)^{1-2 \epsilon}\left(\sin \theta_{n-1}\right)^{1-2 \epsilon} d \theta_{n-1} d \Omega_{2-2 \epsilon}^{n-1} d P_{n-1}^{2} \\
& \ldots \\
& \times(4 \pi)^{2 \epsilon-3}\left(P_{3}^{2}\right)^{-\epsilon}\left(1-\frac{P_{2}^{2}}{P_{3}^{2}}\right)^{1-2 \epsilon}\left(\sin \theta_{2}\right)^{1-2 \epsilon} d \theta_{2} d \Omega_{2-2 \epsilon}^{2} d P_{2}^{2} \\
& \times \frac{(2 \pi)^{2 \epsilon-2}}{8} \frac{\left(p_{\mathrm{T}}^{2}\right)^{-\epsilon}}{\sqrt{P_{2}^{2}}} \sqrt{\frac{P_{2}^{2}}{4}\left(1-\frac{M^{2}}{P_{2}^{2}}\right)^{2}-p_{\mathrm{T}}^{2}}
\end{aligned}
$$

where, in view of the fact that we are interested in the transverse momentum spectrum of the $(n+1)$-th particle $\mathcal{S}(p)$, we have parametrized its phase space in terms of $p_{\mathrm{T}}$ using 
eq. (3.5), while the identification of $p_{T}$ with the transverse momentum in the center-ofmass frame of the hadronic collision, as well as the reason why all other phase spaces are parametrized in terms of $\theta_{i}$ in eq. (3.4), will be clear shortly.

The domain of integration over $p_{\mathrm{T}}^{2}$ in eq. (3.6) is

$$
0 \leq p_{\mathrm{T}}^{2} \leq \frac{P_{2}^{2}}{4}\left(1-\frac{M^{2}}{P_{2}^{2}}\right)^{2},
$$

so that the overall domain of integration eqs. (3.3), (2.24) over the $n$ dimensional variables which characterize the $n$ emissions is ordered, with the upper limit of integration of $p_{T}^{2}$ being set by $P_{2}^{2}$, and then each of the integrations over $P_{i}^{2}$ with $i \geq 2$ being limited from above by $P_{i+1}^{2}$, with the aforementioned identification $P_{n+1}^{2}=\hat{s}$. Of course, in the collinear limit $p_{\mathrm{T}} \rightarrow 0$ this leads to the standard strongly-ordered integration region which leads to collinear factorization and Altarelli-Parisi evolution. This is then the form of the phase space which is most convenient in order to discuss the total inclusive cross section and specifically its soft limit, as was done in refs. [11, 22].

However, in order to consider instead the case in which $p_{\mathrm{T}}$ is kept fixed, the integration region can be re-expressed by taking $p_{\mathrm{T}}$ as outer integration variable. In this case the integration range over $p_{\mathrm{T}}$ is only limited from above according to eq. (2.24), while the integration over all $P_{i}^{2}$ is now in the range

$$
\left(\sqrt{M^{2}+p_{\mathrm{T}}^{2}}+p_{\mathrm{T}}\right)^{2} \leq P_{i}^{2} \leq P_{i+1}^{2}
$$

The final simplification of the phase space eq. (3.6) is achieved by rewriting it in terms of the dimensionless variables

$$
z_{i}=\frac{P_{i}^{2}}{P_{i+1}^{2}}
$$

along with $\tau^{\prime}$ eq. (2.6) and $\xi_{p}$ eq. (2.2). We thus arrive to our final expression for the phase space:

$$
\begin{aligned}
& d \Phi=\tau^{\prime}(4 \pi)^{2 \epsilon-3} Q^{2-2 \epsilon} \tau^{\prime \epsilon-1}\left(1-z_{n}\right)^{1-2 \epsilon}\left(\sin \theta_{n}\right)^{1-2 \epsilon} d \theta_{n} d \Omega_{2-2 \epsilon}^{n} \frac{d z_{n}}{z_{n}} \\
& \times(4 \pi)^{2 \epsilon-3} Q^{2-2 \epsilon}\left(\frac{\tau^{\prime}}{z_{n}}\right)^{\epsilon-1}\left(1-z_{n-1}\right)^{1-2 \epsilon}\left(\sin \theta_{n-1}\right)^{1-2 \epsilon} d \theta_{n-1} d \Omega_{2-2 \epsilon}^{n-1} \frac{d z_{n-1}}{z_{n-1}} \\
& \times(4 \pi)^{2 \epsilon-3} Q^{2-2 \epsilon}\left(\frac{\tau^{\prime}}{z_{n} \ldots z_{3}}\right)^{\epsilon-1}\left(1-z_{2}\right)^{1-2 \epsilon}\left(\sin \theta_{2}\right)^{1-2 \epsilon} d \theta_{2} d \Omega_{2-2 \epsilon}^{2} \frac{d z_{2}}{z_{2}} \\
& \times \frac{(2 \pi)^{2 \epsilon-2}}{4}\left(\frac{\xi_{p}}{\left(\sqrt{1+\xi_{p}}+\sqrt{\xi_{p}}\right)^{2}}\right)^{-\epsilon} \\
& \frac{Q^{2-2 \epsilon}}{\sqrt{\left(1-\frac{\tau^{\prime}}{z_{n} \ldots z_{2}}\right)\left(1-\left(\sqrt{1+\xi_{p}}-\sqrt{\xi_{p}}\right)^{4} \frac{\tau^{\prime}}{z_{n} \ldots z_{2}}\right)}} d \Omega_{2-2 \epsilon}^{1} d \xi_{p} .
\end{aligned}
$$


For fixed $p_{\mathrm{T}}$, the integration over the set of $n-1$ dimensional variables $P_{i}^{2}$ with $2 \leq i \leq n$ now becomes the integral over the $n-1$ variables $z_{i}, 2 \leq i \leq n$. Its range is

$$
\frac{\tau^{\prime}}{z_{n} z_{n-1} \ldots z_{i+1}} \leq z_{i} \leq 1
$$

We note that the phase space eq. (3.10), integrated over the range eq. (3.11) has the structure of a multiple convolution, and thus it factorizes upon taking a Mellin transform with respect to $\tau^{\prime}$, with $n-1$ identical factors depending on momenta $k_{i}, 2 \leq i \leq n$, and one factor depending on the two-body phase space of the leading-order process in which a single parton with momentum $p_{\mathrm{T}}$ recoils against the heavy state $\mathcal{S}(p)$. When comparing to the phase-space factorization of refs. $[11,22]$ it should be kept in mind that eq. (3.10) holds at the differential level in $p_{\mathrm{T}}$ because $\tau^{\prime}$ eq. (2.6) is $p_{\mathrm{T}}$ dependent.

Note that this factorization ensues thanks to the choice of parametrizing momenta $k_{i}$ in terms of the polar angles $\theta_{i}$ : had we chosen to also parametrize them in terms of their transverse component, the Jacobian factors would have spoiled the convolution structure. It is important however to remember that this factorization has been obtained thanks to the choice eq. (3.4)-(3.5) of writing each two-body phase space in the respective centerof-mass frame. Now, in the infrared limit in which the energy of all emitted partons vanishes, all these reference frames coincide: this is the same mechanism which underlies standard CFP factorization [23], and leads to factorization in the eikonal limit [22] if the amplitude factorizes [20]. But for generic momenta, this phase-space factorization is not useful because it only follows by choosing a different reference frame for each emission.

In the threshold limit the squared amplitude has infrared and collinear singularities respectively proportional to $\left(1-z_{i}\right)^{-2}$ and $\left(\sin \theta_{i}\right)^{-2}$, which, when combined with the phase space eq. (3.10), lead each to a simple pole in $\epsilon$ upon integration over the emitted particle's momenta, and are resummed into the Sudakov and soft exponentials of eq. (2.26). Comparing the resummed result eqs. (2.26)-(2.30) to the phase-space eq. (3.10), and noting that the amplitude can only depend polynomially on momenta, we see that the logs resummed into the Sudakov exponent originate from the factor $(1-z)^{-2 \epsilon}$ interfering with the $\epsilon$ poles due to the infrared and collinear integrations over the amplitude. As explained in ref. [22] the fact that the dependence is driven by a phase-space factor reflects its origin from the upper limit of integration on energy of the emitted parton in logarithmically divergent integration.

Furthermore, the logarithmic $\xi_{p}$ dependent factor in the soft anomalous dimension eq. (2.30) is seen to originate from the Jacobian factor $\left(\xi_{p} /\left(\sqrt{1+\xi_{p}}+\sqrt{\xi_{p}}\right)^{2}\right)^{-\epsilon}$ related to the leading-order process: it is thus due to interference between this large-angle radiation, and the $\epsilon$ poles due to soft emission. It is interesting to observe that

$$
\lim _{\xi_{p} \rightarrow 0}\left(\sqrt{1+\xi_{p}}+\sqrt{\xi_{p}}\right)=1,
$$

so logs coming from the soft anomalous dimension eq. (2.30) are absent in the small $p_{\mathrm{T}}$ limit.

On the other hand, in the same limit the Jacobian factor in the denominator of the phase-space related to the leading-order process in the last line of eq. (3.10) becomes

$$
\lim _{\xi_{p} \rightarrow 0} \frac{1}{\sqrt{(1-z)\left(1-\left(\sqrt{1+\xi_{p}}-\sqrt{\xi_{p}}\right)^{4} z\right)}}=\frac{1}{1-z},
$$


thus leading to extra infrared divergences. In fact the resummed Mellin-space transverse momentum distribution for finite $p_{\mathrm{T}}$ in the large- $N$ limit vanishes as $\frac{1}{\sqrt{N}}$. As mentioned in the end of section 2.3, this is the correct large $N$ behaviour of the transverse momentum distribution to any perturbative order. However, if one were to take the $p_{\mathrm{T}} \rightarrow 0$ limit and use the expression on the r.h.s. of eq. (3.13), one would instead find that the transverse momentum distribution grows as $\ln N$; we will come back to this point in section 3.2 below. This shows that the soft and small $p_{\mathrm{T}}$ limits do not commute, as one might expect on physical grounds, given that for finite $p_{T}$ at least one parton must recoil against the colorless final state. Therefore, resummed expressions derived in the $p_{\mathrm{T}} \rightarrow 0$ limit do not provide a fully resummed result for finite $p_{\mathrm{T}}$.

On the other hand, we also note that the threshold and small $p_{\mathrm{T}}$ limits differ, in that the kinematic configurations which contribute to the two limits are different. Indeed, in the threshold limit $s \rightarrow Q^{2}$ the only allowed radiation is that which leaves $Q^{2}$ eq. (2.7) unchanged. This corresponds to radiation of partons which are either infrared, or collinear to the parton recoiling against $\mathcal{S}(p)$. However, the latter collinear radiation does not lead to logarithmic enhancement because $p_{\mathrm{T}}$ is large, and thus does not contribute to the limit. On the other hand, in the small $p_{\mathrm{T}}$ limit one may also have radiation of partons whose transverse momenta are collinear but not soft, in that they are subject to a large boost in the longitudinal direction. It follows that resummed expressions derived for finite $p_{\mathrm{T}}$ do not lead to the correct resummed small $p_{\mathrm{T}}$ limit because not all relevant kinematic configurations are included.

If one wishes to also include collinear non-soft radiation, the phase-space factorization eq. (3.10) does not help because, as mentioned, it only holds choosing a different (boosted) reference frame for each emission. In such case a different phase space factorization must be adopted, as we discuss in the next section.

\subsection{Small $p_{\mathrm{T}}$}

In order to study the small $p_{\mathrm{T}}$ limit we need a factorization of phase space which holds even when longitudinal momenta are not small: this can be done by separating the longitudinal and transverse momentum integrations. Our eventual task is to construct a combined resummed result that reproduces threshold resummation of the total cross section upon integration over transverse momentum. We have seen in the previous section however that threshold resummation at finite $p_{\mathrm{T}}$ does not include collinear contributions, which do contribute to the threshold limit at the integrated level $[2,3]$. We will strive to achieve this phase-space factorization in such a way that the soft limit at the integrated level is not spoiled.

We start from the general form for the phase-space for process eq. (3.1) in $d=4-2 \epsilon$ dimensions:

$$
\begin{aligned}
d \Phi_{n+1}\left(p_{1}, p_{2} ; p, k_{1}, \ldots, k_{n}\right)= & \frac{d^{3-2 \epsilon} p}{(2 \pi)^{3-2 \epsilon} 2 \sqrt{M^{2}+|\vec{p}|^{2}}} \frac{d^{3-2 \epsilon} k_{1}}{(2 \pi)^{3-2 \epsilon} 2 E_{1}} \cdots \frac{d^{3-2 \epsilon} k_{n}}{(2 \pi)^{3-2 \epsilon} 2 E_{n}} \\
& (2 \pi)^{4-2 \epsilon} \delta^{(4-2 \epsilon)}\left(p_{1}+p_{2}-p-k_{1}-\cdots-k_{n}\right) .
\end{aligned}
$$


Representing the transverse momentum constraint as a Fourier transform with respect to an impact parameter $\vec{b}$ conjugate to $\overrightarrow{p_{\mathrm{T}}}$ we get

$$
\begin{gathered}
d \Phi_{n+1}\left(p_{1}, p_{2}, p ; k_{1}, \ldots, k_{n}\right)=(2 \pi)^{2-2 \epsilon} \int d^{2-2 \epsilon} b \frac{|\vec{p}| d|\vec{p}|\left(p_{\mathrm{T}}^{2}\right)^{-\epsilon} d p_{\mathrm{T}}^{2} d \Omega_{2-2 \epsilon} e^{i \vec{b} \cdot \vec{p}_{\mathrm{T}}}}{2 \sqrt{M^{2}+|\vec{p}|^{2}}(2 \pi)^{3-2 \epsilon} \sqrt{|\vec{p}|^{2}-p_{\mathrm{T}}^{2}}} \\
\frac{d E_{1}\left(k_{\mathrm{T}_{1}}^{2}\right)^{-\epsilon} d k_{\mathrm{T}_{1}}^{2} d \Omega_{2-2 \epsilon} e^{i \vec{b} \cdot \vec{k}_{\mathrm{T}_{1}}}}{4(2 \pi)^{3-2 \epsilon} \sqrt{E_{1}^{2}-k_{\mathrm{T}_{1}}^{2}}} \ldots \frac{d E_{n}\left(k_{\mathrm{T}_{\mathrm{n}}}^{2}\right)^{-\epsilon} d k_{\mathrm{T}_{\mathrm{n}}}^{2} d \Omega_{2-2 \epsilon} e^{i \vec{b} \cdot \vec{k}_{\mathrm{T}_{\mathrm{n}}}}}{4(2 \pi)^{3-2 \epsilon} \sqrt{E_{n}^{2}-k_{\mathrm{T}_{\mathrm{n}}}^{2}}} \\
\delta\left(\sqrt{\hat{s}}-\sqrt{M^{2}+|\vec{p}|^{2}}-E_{1}-\cdots-E_{n}\right) \delta\left(p_{z}-k_{1_{z}}-\cdots-k_{n_{z}}\right),
\end{gathered}
$$

where we have also traded the integral over the longitudinal momentum component for an integral over energy.

In order to separate the transverse and longitudinal momentum dependence, it is convenient to adopt a Sudakov parametrization:

$$
\begin{aligned}
k_{1}= & \left(1-z_{1}\right) p_{1}-\frac{k_{\mathrm{T}_{1}}^{2}}{\left(1-z_{1}\right) s} p_{2}+k_{\mathrm{T}_{1}} \\
k_{2}= & z_{1}\left(1-z_{2}\right) p_{1}-\frac{k_{\mathrm{T}_{2}}^{2}}{z_{1}\left(1-z_{2}\right) s} p_{2}+k_{\mathrm{T}_{2}} \\
& \quad \ldots \\
k_{n}= & z_{1} \ldots z_{n-1}\left(1-z_{n}\right) p_{1}-\frac{k_{\mathrm{T}_{\mathrm{n}}}^{2}}{z_{1} \ldots z_{n-1}\left(1-z_{n}\right) s} p_{2}+k_{\mathrm{T}_{\mathrm{n}}} .
\end{aligned}
$$

In the small $p_{\mathrm{T}}$ limit we then get

$$
\begin{aligned}
d \Phi_{n+1}( & \left.p_{1}, p_{2} ; p, k_{1}, \ldots, k_{n}\right)=(2 \pi)^{2-2 \epsilon} \int d^{2-2 \epsilon} b \frac{d|\vec{p}|\left(p_{\mathrm{T}}^{2}\right)^{-\epsilon} d p_{\mathrm{T}}^{2} d \Omega_{2-2 \epsilon} e^{i \vec{b} \cdot \vec{p}_{\mathrm{T}}}}{4 \sqrt{M^{2}+|\vec{p}|^{2}}(2 \pi)^{3-2 \epsilon}} \\
& \frac{d z_{1}\left(k_{\mathrm{T}_{1}}^{2}\right)^{-\epsilon} d k_{\mathrm{T}_{1}}^{2} d \Omega_{2-2 \epsilon} e^{i \vec{b} \cdot \vec{k}_{\mathrm{T}_{1}}}}{4(2 \pi)^{3-2 \epsilon} \sqrt{\left(1-z_{1}\right)^{2}-\frac{4}{s} k_{\mathrm{T}_{1}}^{2}}} \cdots \frac{d z_{n}\left(k_{\mathrm{T}_{\mathrm{n}}}^{2}\right)^{-\epsilon} d k_{\mathrm{T}_{\mathrm{n}}}^{2} d \Omega_{2-2 \epsilon} e^{i \vec{b} \cdot \vec{k}_{\mathrm{T}_{\mathrm{n}}}}}{4(2 \pi)^{3-2 \epsilon} \sqrt{\left(1-z_{n}\right)^{2}-\frac{4}{s z_{1}^{2} \ldots z_{n-1}^{2}} k_{\mathrm{T}_{\mathrm{n}}}^{2}}} \\
& \delta\left(\sqrt{\hat{s}}-\sqrt{M^{2}+|\vec{p}|}-|\vec{p}|\right) \delta\left(|\vec{p}|-\left(1-z_{1} \ldots z_{n}\right) \frac{\sqrt{s}}{2}\right)+\mathcal{O}\left(\frac{1}{b}\right)
\end{aligned}
$$

where we have changed integration variable from $k_{i}$ to $z_{i}$ and we have denoted by $\mathcal{O}\left(\frac{1}{b}\right)$ neglected terms which lead to power suppressed contributions in the small $p_{T}$ limit. Note that we have kept the $k_{\mathrm{T}_{\mathrm{i}}}^{2}$ dependence in the Jacobian square-root factors, even though it is also $\mathcal{O}\left(\frac{1}{b}\right)$, for reasons to be discussed shortly.

The final expression for the phase space is obtained by introducing dimensionless variables $x$, eq. (2.11) and $\xi_{i}=\frac{k_{T, \mathrm{i}}^{2}}{M^{2}}$ defined in analogy to $\xi_{p}$, eq. (2.2), and performing the angular integrations

$$
\int d \Omega_{2-2 \epsilon} e^{i \vec{b} \cdot \overrightarrow{k_{\mathrm{T}}}}=\left(b k_{\mathrm{T}}\right)^{\epsilon}(2 \pi)^{1-\epsilon} J_{-\epsilon}\left(b k_{\mathrm{T}}\right)
$$


where $J_{-\epsilon}$ is implicitly defined by eq. (3.20) and it reduces to the Bessel function $J_{0}$ when $\epsilon \rightarrow 0$. We get

$$
\begin{aligned}
d \Phi_{n+1}\left(p_{1}, p_{2} ; q, k_{1}, \ldots, k_{n}\right)= & x \frac{\pi^{3-2 \epsilon}}{\Gamma(1-\epsilon)} d \xi_{p} \int d b^{2}\left(b p_{\mathrm{T}}\right)^{-\epsilon} b^{n \epsilon} J_{-\epsilon}\left(b p_{\mathrm{T}}\right) \\
& J_{-\epsilon}\left(b k_{\mathrm{T}_{1}}\right) \frac{M^{-\epsilon}\left(\xi_{1}\right)^{-\frac{\epsilon}{2}} d z_{1} d \xi_{1}}{4(2 \pi)^{2-\epsilon} \sqrt{\left(1-z_{1}\right)^{2}-4 x \xi_{1}}} \\
& \ldots \\
& J_{-\epsilon}\left(b k_{\mathrm{T}_{\mathrm{n}}}\right) \frac{M^{-\epsilon}\left(\xi_{n}\right)^{-\frac{\epsilon}{2}} d z_{n} d \xi_{n}}{4(2 \pi)^{2-\epsilon} \sqrt{\left(1-z_{n}\right)^{2}-\frac{4 x}{z_{1}^{2} \ldots z_{n-1}^{2}} \xi_{n}}} \\
& \times \delta\left(x-z_{1} \ldots z_{n}\right)+\mathcal{O}\left(\frac{1}{b}\right) .
\end{aligned}
$$

The integration range over transverse momenta is

$$
0 \leq \xi_{i} \leq \frac{z_{1}^{2} \ldots z_{i-1}^{2}\left(1-z_{i}\right)^{2}}{4 x}
$$

while all $z_{i}$ range from $0 \leq z_{i} \leq 1$.

The expression of the phase space eq. (3.21) would have the structure of a convolution, and thus factorize upon Mellin transformation with respect to $x$, were it not for the $\xi_{i}$ terms in the denominator. Up to $\mathcal{O}\left(\frac{1}{b}\right)$ corrections, these can be simplified by letting all $\xi_{i} \rightarrow 0$. This then leads to a factorized form of phase space which, when combined with a suitably factorized and renormalization-group improved form of the amplitude, leads to transverse momentum resummation eq. (2.31). However, this also leads to a result which in the soft limit does not have have the correct behaviour and is beset by a spurious logarithmic rise, because of the non-commutativity of limits which we have already seen in eq. (3.13) when discussing the factorization of phase space in the soft limit. We will now show first, how transverse momentum resummation is usually derived in the $\xi_{i} \rightarrow 0$ limit, then, why this expansion spoils the soft limit at the integrated level, and finally, how factorization of phase space can be obtained while preserving the correct soft limit.

The $\xi_{i} \rightarrow 0$ limit of eq. (3.21) must be taken with some care because new infrared singularities arise in the limit. These can be taken care of by rewriting the Jacobian square-root factors as

$$
\begin{aligned}
\frac{1}{\sqrt{\left(1-z_{1}\right)^{2}-4 x \xi_{n}}}= & {\left[\frac{1}{\sqrt{\left(1-z_{1}\right)^{2}-4 x}}\right]_{+}^{z_{1}} } \\
& +\frac{1}{2} \ln \frac{1-\sqrt{1-4 x \xi_{1}}}{1+\sqrt{1-4 x \xi_{1}}} \delta\left[z_{1}-\left(1-2 \sqrt{x \xi_{n}}\right)\right],
\end{aligned}
$$

and similarly for all $z_{i}$, where the plus distribution is defined as

$$
\int_{0}^{1} d z g(z)[f(z)]_{+}^{z}=\int_{0}^{1} d z[g(z)-g(1)] f(z) .
$$


Substituting eq. (3.23) in eq. (3.21) we can now safely take the $\xi_{i} \rightarrow 0$ limit, with the result

$$
\begin{aligned}
d \Phi_{n+1}\left(p_{1}, p_{2} ; q, k_{1}, \ldots, k_{n}\right)= & x \frac{\pi^{3-2 \epsilon}}{\Gamma(1-\epsilon)} d \xi_{p} \int d b^{2}\left(b p_{\mathrm{T}}\right)^{-\epsilon} b^{n \epsilon} J_{-\epsilon}\left(b p_{\mathrm{T}}\right) \\
& J_{-\epsilon}\left(b k_{\mathrm{T}_{1}}\right) \frac{M^{-\epsilon}\left(\xi_{1}\right)^{-\frac{\epsilon}{2}}}{4(2 \pi)^{2-\epsilon}}\left[\left[\frac{1}{1-z_{1}}\right]_{+}-\delta\left(1-z_{1}\right) \frac{1}{2} \ln \xi_{1}\right] d z_{1} d \xi_{1} \\
& \ldots \\
& J_{-\epsilon}\left(b k_{\mathrm{T}_{\mathrm{n}}}\right) \frac{M^{-\epsilon}\left(\xi_{n}\right)^{-\frac{\epsilon}{2}}}{4(2 \pi)^{2-\epsilon}}\left[\left[\frac{1}{1-z_{n}}\right]_{+}-\delta\left(1-z_{n}\right) \frac{1}{2} \ln \xi_{n}\right] d z_{n} d \xi_{n} \\
& \times \delta\left(x-z_{1} \ldots z_{n}\right)+\mathcal{O}\left(\frac{1}{b}\right)
\end{aligned}
$$

where now the integration range over transverse momenta is

$$
0 \leq \xi_{i} \leq \infty
$$

for all $\xi_{i}$.

The phase space eq. (3.25) factorizes upon Mellin transform. It can be used to derive the standard transverse momentum resummation eq. (2.32): in the small $p_{\mathrm{T}}$ limit the squared amplitude has collinear singularities (both soft and non-soft) which are resummed into the Sudakov form factor eq. (2.32), with the $A^{p_{\mathrm{T}}}\left(\alpha_{s}\right)$ term driven by the interference with the infrared singularity of the DGLAP anomalous dimension with the contributions to the phase space eq. (3.25) enhanced by $\ln \xi_{i}$.

However, this resummed expression, eq. (2.31), does not reproduce the correct behaviour of the total cross section in the soft limit upon integration over $p_{\mathrm{T}}$. This can be seen by noting that, integrating over the phase space eq. (3.21) in the range eq. (3.22) and expanding in powers of $\xi_{i}$, one ends up with integrals (one for each emission) of the form

$$
\begin{aligned}
\int_{0}^{\frac{(1-z)^{2}}{4}} d \xi \frac{1}{\sqrt{(1-z)^{2}-4 \xi}} & =\frac{1}{1-z} \int_{0}^{\frac{(1-z)^{2}}{4}} d \xi 1+\frac{2 \xi}{(1-z)^{2}}+\frac{6 \xi^{2}}{(1-z)^{4}}+\ldots \\
& =\frac{(1-z)}{4}\left(1+\frac{1}{4}+\frac{1}{8}+\ldots\right)
\end{aligned}
$$

where for definiteness we have considered integration with respect to $\xi_{1}$. It is apparent that all terms in the expansion in powers of $\xi_{i}$ are in fact of the same order after integration. Hence, only retaining the first term in this expansion as it was done in the derivation of eq. (3.25) spoils the $x \rightarrow 1$ limit at the integrated level.

The relevant power counting is clear in Fourier-Mellin space, where resummed expressions are derived, and in which the expansion eq. (3.27) becomes a series in powers of $\frac{1}{b}$ :

$$
\int_{0}^{1} d z z^{N-1} \int_{0}^{\frac{(1-z)^{2}}{4}} d \xi J_{0}(b M \sqrt{\xi}) \frac{1}{\sqrt{(1-z)^{2}-4 \xi}}=\frac{2}{b^{2} M^{2}}\left(1-\frac{4 N^{2}}{b^{2} M^{2}}+\frac{16 N^{4}}{b^{4} M^{4}}+\ldots\right) .
$$


Namely, it is clear that the expansion parameter is $\frac{N}{b}$. Because the inclusive cross section is obtained by taking $b=0$, truncating this expansion spoils the large $N$ behaviour at the inclusive level. Hence, in order to preserve the threshold limit at the inclusive level, transverse momentum resummation must be performed by taking the limit $b \rightarrow \infty$ at fixed $\frac{N}{b}$, rather than at fixed $N$.

A form of the phase space which does factorize upon Mellin transform, but which does not spoil the soft limit at the integrated level can be obtained by evaluating the phase space eq. (3.21) and integration range eq. (3.22) in the soft limit, namely

$$
\begin{aligned}
d \Phi_{n+1}\left(p_{1}, p_{2} ; q, k_{1}, \ldots, k_{n}\right)= & x \frac{\pi^{3-2 \epsilon}}{\Gamma(1-\epsilon)} d \xi_{p} \int d b^{2}\left(b p_{\mathrm{T}}\right)^{-\epsilon} b^{n \epsilon} J_{-\epsilon}\left(b p_{\mathrm{T}}\right) \\
& J_{-\epsilon}\left(b k_{\mathrm{T}_{1}}\right) \frac{M^{-\epsilon}\left(\xi_{1}\right)^{-\frac{\epsilon}{2}} d z_{1} d \xi_{1}}{4(2 \pi)^{2-\epsilon} \sqrt{\left(1-z_{1}\right)^{2}-4 z_{1} \xi_{1}}} \\
& \ldots \\
& J_{-\epsilon}\left(b k_{\mathrm{T}_{\mathrm{n}}}\right) \frac{M^{-\epsilon}\left(\xi_{n}\right)^{-\frac{\epsilon}{2}} d z_{n} d \xi_{n}}{4(2 \pi)^{2-\epsilon} \sqrt{\left(1-z_{n}\right)^{2}-4 z_{n} \xi_{n}}} \\
& \times \delta\left(x-z_{1} \ldots z_{n}\right)+\mathcal{O}\left(\frac{1}{b}\right)+\mathcal{O}\left(\frac{1}{N}\right)
\end{aligned}
$$

and

$$
0 \leq \xi_{i} \leq \frac{\left(1-z_{i}\right)^{2}}{4 z_{i}}
$$

where we have denoted by $\mathcal{O}\left(\frac{1}{N}\right)$ terms which do not contribute to the threshold limit. In going from eq. (3.21) to eq. (3.29) we have retained the unexpanded phase-space factor of eq. (3.28), but in it we have neglected the factors of $\frac{1}{z_{i}^{2}}$ which multiply $\xi$, which would spoil the convolution structure, but correct it by $\mathcal{O}\left(\frac{1}{N}\right)$ terms. As a consequence, the result now has the form of a convolution, and it factorizes upon Mellin transform when combined with an amplitude that also has the structure of a convolution, but now it also includes all the contributions in the phase space at large $b$ of $\mathcal{O}\left(\frac{N^{k}}{b^{k}}\right)$.

Recalling that transverse momentum distributions are convolutive with respect to the partonic scaling variable $x$ eq. (2.11), one has

$$
\begin{aligned}
& \int_{0}^{1} d x x^{N-1} \int d \xi_{1} d \xi_{2} \ldots d \xi_{n} \frac{d \Phi_{n+1}\left(p_{1}, p_{2} ; q, k_{1}, \ldots, k_{n}\right)}{d \xi_{p}}= \\
& =\frac{\pi^{2-\epsilon} b^{n \epsilon}}{\Gamma(1-\epsilon)} \int d b^{2}\left(b p_{\mathrm{T}}\right)^{-\epsilon} J_{-\epsilon}\left(b p_{\mathrm{T}}\right)\left(\sqrt{1+\xi_{p}}-\sqrt{\xi_{p}}\right)^{-2 N} \\
& {\left[\int_{0}^{\infty} d \xi(\sqrt{1+\xi}-\sqrt{\xi})^{2 N} J_{-\epsilon}\left(b k_{\mathrm{T}}\right) M^{-\epsilon}(\xi)^{-\frac{\epsilon}{2}}\right.} \\
& \left.\int_{0}^{1} d z z^{N-1} \frac{1}{4(2 \pi)^{2-\epsilon} \sqrt{(1-z)\left(1-(\sqrt{1+\xi}-\sqrt{\xi})^{4} z\right)}}\right]^{n}+\mathcal{O}\left(\frac{1}{b}\right)+\mathcal{O}\left(\frac{1}{N}\right)
\end{aligned}
$$


where we have used eq. (2.11), and we have interchanged the $\xi_{i}$ and $z_{i}$ integrations and performed the change of integration variables $z_{i} \rightarrow z_{i}^{\prime}\left(\sqrt{1+\xi_{i}}-\sqrt{\xi_{i}}\right)^{2}$.

It is interesting to observe that, when combined with a matrix element which behaves as a constant when $z_{i} \rightarrow 1$, the phase space eq. (3.31) leads to the correct large- $N$ behaviour of the leading-order transverse momentum distribution, which, as discussed in section 2.3, is $\frac{d \hat{\sigma}_{i j}^{\mathrm{LO}}}{d \xi_{p}}\left(N, \xi_{p}\right) \underset{N \rightarrow \infty}{\sim} \frac{1}{\sqrt{N}}$. Indeed, at leading order, $n=1$ and $\xi_{p}=\xi$ in eq. (3.31), so the Mellin transform of the transverse momentum distribution behaves as

$$
\int_{0}^{1} d z z^{N-1} \frac{1}{\sqrt{(1-z)\left(1-\left(\sqrt{1+\xi_{p}}-\sqrt{\xi_{p}}\right)^{4} z\right)}}=\frac{\sqrt{\pi}}{2 \sqrt{N}}\left(\left(\frac{\xi_{p}}{1+\xi_{p}}\right)^{\frac{1}{4}}+\left(\frac{1+\xi_{p}}{\xi_{p}}\right)^{\frac{1}{4}}\right)+\mathcal{O}\left(\frac{1}{N}\right) .
$$

Had the form eq. (3.25) of the phase space been used instead, one would take $\xi_{1}=0$ first, and find a Mellin-space transverse momentum distribution which displays a spurious logarithmic growth

$$
\int_{0}^{1} d z z^{N-1}\left[\frac{1}{1-z}\right]_{+}=-\ln N+\mathcal{O}(1)
$$

This is another manifestation of the fact that truncation of the expansion eq. (3.27) spoils the soft limit, because higher order terms are enhanced by powers of $1-x$ despite being suppressed by powers of $\xi_{i}$

\section{Resummation}

Using the phase-space arguments of section 3 we can now construct a combined resummed expression which satisfies all of the requirements discussed in the introduction. First, in section 4.1 we will present a formal construction of our resummed formula and argue that it has the required properties, then, in section 4.2 we will provide explicit expressions in the case of Higgs production in gluon fusion up to NNLL, and check explicitly that in the three limits of large $N$ at fixed $p_{\mathrm{T}}$, small $p_{\mathrm{T}}$ for generic $N$, and large $N$ at the integrated level, known NNLL results are reproduced. This will provide us with nontrivial insight on the relation between these different resummations.

Here and henceforth will refer to the $N \rightarrow \infty$ or $x \rightarrow 1$ limit as threshold limit. Resummation in this limit, which we will refer to as threshold resummation, includes, to a given logarithmic order and to all orders in $\alpha_{s}$, powers of $\ln N$ or $\ln (1-x)$, up to contributions which have a relative power suppression in $1 / N$ or $1-x$. Unless otherwise stated, threshold resummation will refer to the resummation being performed at fixed and finite $p_{\mathrm{T}}$. We will refer to the $b \rightarrow \infty$ or $p_{\mathrm{T}} \rightarrow 0$ limit as small $p_{\mathrm{T}}$ limit. Resummation in this limit, which we will refer to as transverse momentum resummation, includes, to a given logarithmic order, and to all orders in $\alpha_{s}$, powers of $\ln b$ or $\ln \xi_{p}$, up to contributions which have a relative power suppression in $1 / b$ or $\xi_{p}$.

\subsection{The combined resummed result}

The combined resummed result can be constructed based on two sets of observations, which substantially rely on the phase-space analysis of section 3 . 
The first concerns the relation between transverse momentum resummation and threshold resummation at finite $p_{\mathrm{T}}$. The key observation is that the threshold limit and the small $p_{\mathrm{T}}$ limit do not commute, eqs. (3.12)-(3.13). It then follows that because of eq. (3.13) [see also eqs. (3.32)-(3.33)], results derived in the small $p_{\mathrm{T}}$ limit display an unphysical growth with $N$ in the threshold limit. However, this unphysical growth can be removed, and the correct large $N$ behaviour for finite $p_{\mathrm{T}}$ can be restored, if the form eq. (3.31) of phase-space is used in the derivation of transverse momentum resummation, instead of the usual one eq. (3.25). This form of the phase space is compatible with factorization leading to transverse momentum resummation, and differs from the standard result by terms which are power-suppressed in the small $p_{\mathrm{T}}$ limit: it thus leads to an alternative form of transverse momentum resummation which differs from the usual one by power-suppressed terms $\mathcal{O}\left(\frac{1}{b}\right)$ at fixed $N$.

However, even after doing this, because of eq. (3.12), results derived in the small $p_{\mathrm{T}}$ limit do not contain all of the large $N$ logs which are present at finite $p_{\mathrm{T}}$. Furthermore, while soft radiation (which is responsible for logs in the threshold limit) is also collinear, collinear radiation (which is responsible for logs in the small $p_{\mathrm{T}}$ limit) can be non-soft: as a consequence, transverse momentum resummation includes logarithmic contributions that do not contribute to the soft limit.

The upshot is that transverse momentum resummation in the soft limit displays an unphysical growth with $N$ which may be removed by inclusion of terms which are powersuppressed in the small $p_{\mathrm{T}}$ limit, but even having done that, transverse momentum resummation in the large $N$ limit still does not include all large logs which contribute to the threshold limit of the full cross section and are included in threshold resummation. Conversely, threshold resummation in the small $p_{\mathrm{T}}$ limit does not include all large logs which contribute to the small $p_{\mathrm{T}}$ limit of the full cross section and are included in transverse momentum resummation.

The second set of observations concerns the relation between resummation of the transverse momentum distribution, and the threshold limit of the inclusive cross section after integration over transverse momentum. Here the key observation is that in order to be enhanced in the threshold limit after integration over transverse momentum, a contribution to the amplitude must have the highest allowed power growth as $p_{\mathrm{T}} \rightarrow 0$, otherwise integration over transverse momentum leads to a contribution which is power-suppressed in the soft limit. It then follows that the full threshold limit at the inclusive level can be obtained from integration over $p_{\mathrm{T}}$ of all terms which contribute to transverse momentum resummation. However, transverse momentum resummation must be performed in the soft limit, i.e., retaining in the resummed expressions all terms which are enhanced in the threshold limit even though they might be suppressed in the small $p_{\mathrm{T}}$ limit. In FourierMellin space this means that we must retain the leading-order contribution in the large $b$ limit at $\frac{N}{b}$ fixed, rather than at fixed $N$ as it is usually done. This specifically implies that we must use the form eq. (3.31) of phase-space, instead of the usual one eq. (3.25), because of eqs. (3.27), (3.28).

Based on this observation, a combined resummed expression which satisfies all requirements can be constructed in two steps. First, we construct a modified version $\frac{d \hat{\sigma}_{i j}^{\mathrm{tr}}}{d \xi_{p}}$ 
of transverse momentum resummation $\frac{d \hat{\sigma}_{i j}^{\text {tr }}}{d \xi_{p}}$ eq. (4.1), in which transverse momentum resummation is performed in the soft limit, i.e. expanding in powers of $\frac{1}{b}$ at fixed $\frac{N}{b}$ as just explained. This is obtained by noting that the squared amplitude for real emission behaves as $|A|^{2} \underset{\xi_{i} \rightarrow 0}{\sim} \xi_{i}^{-1}$ as $\xi_{i} \rightarrow 0$, which leads to the infrared and collinear singularities resummed in eq. (2.31), with infrared singularities canceled by virtual terms. Unlike the phase-space measure eq. (3.21), the square amplitude does not display further infrared singularities as $x \rightarrow 1$ because standard power counting arguments ensure that all infrared singularities in propagators arise in the collinear if $p_{\mathrm{T}} \rightarrow 0$ limit. Hence the only $\frac{N}{b}$ terms arise due to the phase space, and the amplitude can be safely expanded in powers of $\xi_{p}$ at fixed $N$. It is then sufficient to modify the standard transverse momentum resummation eq. (2.31) in order to account for the use of the phase space eqs. (3.29)-(3.30) instead of the standard phase space eqs. (3.25)-(3.22)).

Using the Mellin-space expression eq. (3.31) we then get

$$
\begin{aligned}
& \frac{d \hat{\sigma}_{i j}^{\operatorname{tr}^{\prime}}}{d \xi_{p}}\left(N, \xi_{p}, \alpha_{s}\left(M^{2}\right), M^{2}\right)=\sigma_{0} \int_{0}^{\infty} d b \frac{b}{2} J_{0}\left(b M \sqrt{\xi_{p}}\right)\left(\sqrt{1+\xi_{p}}-\sqrt{\xi_{p}}\right)^{-2 N} \\
& \mathcal{H}_{i j}\left(N, \alpha_{s}\left(M^{2}\right)\right) \exp \left[\int_{0}^{\infty} d \xi(\sqrt{1+\xi}-\sqrt{\xi})^{2 N} J_{0}(b M \sqrt{\xi})\left[\frac{\mathcal{B}\left(N, \alpha_{s}\left(M^{2} \xi\right)\right)}{\xi}\right]_{+}^{p_{\mathrm{T}}}+\mathcal{O}\left(\frac{1}{b}\right)\right] \\
& \exp \left[\int_{0}^{\infty} d \xi(\sqrt{1+\xi}-\sqrt{\xi})^{2 N} J_{0}(b M \sqrt{\xi}) \int_{0}^{1} d z z^{N-1}\right. \\
& \left(\left[\frac{2 A^{p_{\mathrm{T}}}\left(\alpha_{s}\left(M^{2} \xi\right)\right)}{\xi}\right]_{+}^{p_{\mathrm{T}}}\left[\frac{1}{\sqrt{(1-z)\left(1-(\sqrt{1+\xi}-\sqrt{\xi})^{4} z\right)}}\right]_{+}^{z}+\delta(1-z) \frac{1}{2(\sqrt{1+\xi}-\sqrt{\xi})^{2}}\right. \\
& \left(2 A^{p_{\mathrm{T}}}\left(\alpha_{s}\left(M^{2} \xi\right)\right) \frac{\ln (1+\xi)}{\xi}-\left[\frac{2 A^{p_{\mathrm{T}}}\left(\alpha_{s}\left(M^{2} \xi\right)\right) \ln \xi}{\xi}\right]_{+}^{p_{\mathrm{T}}}\right) \\
&
\end{aligned}
$$

Here we have defined a second plus distribution

$$
\int_{0}^{1} d \xi g(\xi)[f(\xi)]_{+}^{p_{\mathrm{T}}}=\int_{0}^{1} d \xi[g(\xi)-g(0)] f(\xi)
$$

along with the usual one eq. (3.24); note that the integration range in eq. (4.2) is not the same as the integration range in eq. (4.1). In order to get from eqs. (2.31), (3.31) to eq. (4.1) we have used the identity

$$
\begin{aligned}
\frac{1}{\sqrt{(1-z)\left(1-(\sqrt{1+\xi}-\sqrt{\xi})^{4} z\right)}}= & {\left[\frac{1}{\sqrt{(1-z)\left(1-(\sqrt{1+\xi}-\sqrt{\xi})^{4} z\right)}}\right]_{+}^{z} } \\
& +\delta(1-z) \frac{1}{2(\sqrt{1+\xi}-\sqrt{\xi})^{2}}[\ln (1+\xi)-\ln \xi],
\end{aligned}
$$


and we have further assumed cancellation of infrared singularities, so all explicit $\frac{1}{\xi}$ terms are replaced by the corresponding plus distributions.

The notation $\mathcal{O}\left(\frac{1}{b}\right)$ in eq. (4.1) means that all quantities are evaluated at leading order in an expansion in powers of $b$ for fixed $\frac{N}{b}$, as discussed above. The function $A^{p_{\mathrm{T}}}$ is then the same as in the standard transverse momentum resummation eq. (2.31), while $\mathcal{B}$, which contains also contributions not enhanced as $N \rightarrow \infty$, has to be determined by matching to the function $B$ of eq. (2.31). Clearly, just like the function $B$ of eq. (2.31), also $\mathcal{B}$ is a matrix on the flavour space and the first exponential of eq. (4.1) must be viewed as path-ordered. Finally, the function $\mathcal{H}_{i j}\left(N, \alpha_{s}\right)$ is determined by first, matching it to a fixed order calculation, just like the function $H_{i j}\left(N, \alpha_{s}\right)$ of eq. (2.31) was (or, equivalently, matching $\mathcal{H}_{i j}\left(N, \alpha_{s}\right)$ to $\left.H_{i j}\left(N, \alpha_{s}\right)\right)$ and then, by observing that in order to fully reproduce threshold resummation at the integrated level, this function must be resummed to all orders in $\alpha_{s}$ in the threshold limit up to the desired logarithmic order in $\ln N$, rather than just computed to finite order. Explicitly, we let

$$
\mathcal{H}_{i j}\left(N, \alpha_{s}\left(M^{2}\right)\right)=\mathcal{H}_{i j}^{\text {f.o. }}\left(N, \alpha_{s}\left(M^{2}\right)\right)+\mathcal{H}_{i j}^{0}\left(\alpha_{s}\left(M^{2}\right)\right) \exp \left[-D^{p_{\mathrm{T}}}\left(\alpha_{s}\left(M^{2}\right)\right) \ln N\right] .
$$

The expression eq. (4.1) automatically leads to threshold resummation of the cross section upon integration over transverse momentum, and it reproduces the correct physical behaviour in the soft limit for finite $p_{\mathrm{T}}$. However, it does not contain all logarithmically enhanced terms in the soft limit for finite $p_{\mathrm{T}}$. In order to achieve full threshold resummation for finite $p_{\mathrm{T}}$, we combine $\frac{d \hat{\sigma}_{i j}^{\mathrm{tr}^{\prime}}}{d \xi_{p}}$ with the standard threshold resummation $\frac{d \hat{\sigma}_{j}^{\text {th }}}{d \xi_{p}}$ eq. (2.26) in a way which avoids double counting. This can be done by introducing a matching function $T\left(N, \xi_{p}\right)$ such that

$$
\begin{aligned}
& \lim _{N \rightarrow \infty} T\left(N, \xi_{p}\right)=1 \\
& \lim _{\xi_{p} \rightarrow 0} T\left(N, \xi_{p}\right)=0,
\end{aligned}
$$

and letting

$$
\begin{aligned}
\frac{d \hat{\sigma}_{i j}}{d \xi_{p}}\left(N, \xi_{p}, \alpha_{s}\left(\mu_{R}^{2}\right), \mu_{F}^{2}\right)= & \left(1-T\left(N, \xi_{p}\right)\right) \frac{d \hat{\sigma}_{i j}^{\mathrm{tr}^{\prime}}}{d \xi_{p}}\left(N, \xi_{p}, \alpha_{s}\left(\mu_{R}^{2}\right), \mu_{F}^{2}\right) \\
& +T\left(N, \xi_{p}\right) \frac{d \hat{\sigma}_{i j}^{\mathrm{th}}}{d \xi_{p}}\left(N, \xi_{p}, \alpha_{s}\left(\mu_{R}^{2}\right), \mu_{F}^{2}\right) .
\end{aligned}
$$

Clearly, this avoids double-counting of any term in common between $\frac{d \hat{\sigma}_{i j}^{\text {tr' }}}{d \xi_{p}}$ and $\frac{d \hat{\sigma}_{i j}^{\text {th }}}{d \xi_{p}}$. Furthermore, because of the limits eqs. (4.5)-(4.6), the combined result eq. (4.7) reproduces threshold resummation for large $N$ and transverse momentum resummation for small $\xi_{p}$, up to corrections whose size can be tuned by choosing the form of the matching function. For instance choosing

$$
T\left(N, \xi_{p}\right)=\frac{N^{k} \xi_{p}^{m}}{1+N^{k} \xi_{p}^{m}}
$$

the combined result differs from $\frac{d \hat{\sigma}_{j}^{\mathrm{tr}^{\prime}}}{d \xi_{p}}$ by $\mathcal{O}\left(\xi_{p}^{m}\right)$ corrections when $\xi_{p} \rightarrow 0$, and from $\frac{d \hat{\sigma}_{i j}^{\mathrm{th}}}{d \xi_{p}}$ by $\mathcal{O}\left(\frac{1}{N^{k}}\right)$ corrections when $N \rightarrow \infty$. 
Note that transverse momentum resummation eq. (4.1) is most easily performed by choosing $\mu_{R}^{2}=\mu_{F}^{2}=M^{2}$, and threshold resummation eq. (2.26) by choosing $\mu_{R}^{2}=\mu_{F}^{2}=Q^{2}$ as given by eq. (2.7); however the combined expression eq. (4.7) must be written with a common choice of renormalization and factorization scale after evolving either or both of its two terms to a common scale using standard renormalization group expressions.

Equation (4.7) reproduces by construction threshold resummation $\frac{d \hat{\sigma}_{i j}^{\text {th }}}{d \xi_{p}}$ eq. $(2.26)$ in the large- $N$ limit at fixed $\xi_{p}$, and transverse momentum resummation $\frac{d \hat{\sigma}_{i j}^{\operatorname{tr}^{\prime}}}{d \xi_{p}}$ eq. (4.1) in the $p_{\mathrm{T}} \rightarrow 0$ limit at fixed $N$, because of eq. (4.5) satisfied by the matching function $T\left(N, \xi_{p}\right)$. Furthermore, the form of the matching function also ensures that the threshold resummation contribution $\frac{d \hat{\sigma}_{i j}^{\text {th }}}{d \xi_{p}}$ does not contribute to the threshold limit of the total cross section, because in the $\xi_{p} \rightarrow 0$ limit it is regular and thus upon integration it is free of logarithmic enhancement. On the other hand, the small- $\xi_{p}$ logarithmic singularities of eq. (4.1) coincide to all logarithmic orders with those of the transverse momentum resummation contribution $\frac{d \hat{\sigma}_{j}^{\mathrm{rt}^{\prime}}}{d \xi_{p}}$. Hence, upon integration over transverse momentum, the combined result $\frac{d \hat{\sigma}_{i j}}{d \xi_{p}}$ has a threshold limit which coincides with that of the integral of $\frac{d \hat{\sigma}_{i j}^{\prime}}{d \xi_{p}}$, and thus it reproduces the threshold limit of the total cross section for the reasons explained.

We conclude that eq. (4.7) satisfies all requirements spelled out in the introductory section 1, and thus we will take it as our final combined resummed expression. In the next section we will work it out in a fully explicit way for the case of Higgs production in gluon fusion, and verify that indeed it has all its desired properties. In the course of this argument, we will work out a more manageable form of our resummed result.

\subsection{Higgs production}

We will now work out explicitly our combined resummed expression eqs. (4.7) for the case of Higgs production in gluon fusion up to NNLL accuracy. This will allow us to explicitly check that indeed our result has its desired properties. In the process of doing this, we will derive a simpler, closed-form expression for our resummed result by suitably neglecting subleading terms. Our starting point is the expression of threshold resummation $\frac{d \hat{\sigma}_{i j}^{\text {th }}}{d \xi_{p}}$ eq. (2.26) and transverse momentum resummation $\frac{d \hat{\sigma}_{i j}^{\mathrm{tr}^{\prime}}}{d \xi_{p}}$ eq. (4.1). Note that in the $p_{\mathrm{T}} \rightarrow 0$ limit the cross section starts at $\mathcal{O}\left(\alpha_{s}^{0}\right)$ (in the effective field theory limit, corresponding to $\mathcal{O}\left(\alpha_{s}^{2}\right)$ in full QCD with heavy quark masses), while for finite $p_{\mathrm{T}}$ the transverse momentum distribution starts at $\mathcal{O}\left(\alpha_{s}\right)$, hence there is a mismatch in counting fixed or resummed order: e.g. a $\mathrm{N}^{k} \mathrm{LO}$ contribution to the transverse momentum spectrum is a $\mathrm{N}^{k+1} \mathrm{LO}$ contribution to the $p_{\mathrm{T}} \rightarrow 0$ (or inclusive) cross section. However, because of the way our combined resummed expression eq. (4.7) is constructed there is no harm in including either of the two contributions to it at an extra perturbative order. Note that two different definitions of logarithmic order exist in the literature, according to whether one counts orders in the exponent, or for the expanded cross section; we will follow the nomenclature given e.g. in table 1 of ref. [24]. 
We will present results up to NNLL accuracy for transverse momentum resummation and to NNLL* for threshold resummation at finite $p_{\mathrm{T}}$, which, upon integration over $\xi_{p}$ reproduce NNLL* threshold resummation for the inclusive cross section. Explicit values of the coefficients needed in order to achieve this accuracy are collected in appendices A.1-A.2, while in appendix A.3 we collect the coefficients which are needed in order to check that inclusive threshold resummation is reproduced. For transverse momentum resummation, they are obtained matching our generalized resummed result $\frac{d \hat{\sigma}_{i j}^{\mathrm{tr}^{\prime}}}{d \xi_{p}}$ eq. (4.1) to the NNLL result of refs. [17, 25]. For threshold resummation they are obtained by extending up to NNLL* the NLL expression of ref. [4].

As already mentioned, eq. (4.7) reproduces by construction threshold resummation in the large- $N$ limit at fixed $\xi_{p}$, and transverse momentum resummation eq. (4.1) in the $p_{\mathrm{T}} \rightarrow 0$ limit at fixed $N$ and it reproduces the threshold limit of the total cross section upon integration over $p_{\mathrm{T}}$ if the modified transverse momentum resummation $\frac{d \hat{\sigma}_{i j}^{\mathrm{tr}^{\prime}}}{d \xi_{p}}$ does. It thus remains to work out the explicit expression for the contribution $\frac{d \hat{\sigma}_{j}^{\mathrm{tr}^{\prime}}}{d \xi_{p}}$ eq. (4.1), and check that computing it with the values of the coefficients listed in appendix A.2, such that it gives standard transverse momentum resummation eq. (2.32) in the $p_{\mathrm{T}} \rightarrow 0$ limit, it indeed leads to threshold resummation of the total cross section upon integration over $p_{\mathrm{T}}$. As a byproduct of this check, we will end up with an explicit closed-form expression for the combined resummed result.

We start from the resummed expression eq. (4.1). As discussed above, the exponent of this expression ought to be evaluated at leading order in an expansion in powers of $\frac{1}{b}$ at fixed $\frac{N}{b}$. As a consequence of our combined resummation formalism, this expression, as a function of $N$ and $b$, reduces to standard transverse momentum resummation if expanded to leading order in powers of $b$ at fixed $N$, while for $b=0$ it reproduces the total cross section in the threshold limit $N \rightarrow \infty$.

Expanding $A^{p_{\mathrm{T}}}$ and $\mathcal{B}$ in powers of $\alpha_{s}$ and expressing the result in terms of $\alpha_{s}\left(M^{2}\right)$ all integrals in the exponent are of the form

$$
\begin{aligned}
G_{k, 1}(N, b)= & \int_{0}^{\infty} d \xi(\sqrt{1+\xi}-\sqrt{\xi})^{2 N} J_{0}(b M \sqrt{\xi}) \int_{0}^{1} d z z^{N-1} \\
& \left(\left[\frac{\ln ^{k} \xi}{\xi}\right]_{+}^{p_{\mathrm{T}}}\left[\frac{1}{\sqrt{(1-z)\left(1-(\sqrt{1+\xi}-\sqrt{\xi})^{4} z\right)}}\right]_{+}^{z}+\delta(1-z) \frac{1}{2(\sqrt{1+\xi}-\sqrt{\xi})^{2}}\right. \\
& \left.\left(\frac{\ln (1+\xi) \ln ^{k} \xi}{\xi}-\left[\frac{\ln ^{k+1} \xi}{\xi}\right]_{+}^{p_{\mathrm{T}}}\right)\right) \\
G_{k, 2}(N, b)= & \int_{0}^{\infty} d \xi(\sqrt{1+\xi}-\sqrt{\xi})^{2 N} J_{0}(b M \sqrt{\xi})\left[\frac{\ln ^{k} \xi}{\xi}\right]_{+}^{p_{\mathrm{T}}},
\end{aligned}
$$

where the integrals $G_{k, 1}$ and $G_{k, 2}$ appear in the terms proportional to $A^{p_{\mathrm{T}}}$ and $\mathcal{B}$ respectively. We need to evaluate these integrals in the limit $b \rightarrow \infty$ at fixed $\frac{N}{b}$. 
This can be done by defining two generating functions, $\mathcal{G}_{1}(N, b, \epsilon)$ and $\mathcal{G}_{2}(N, b, \epsilon)$ such that

$$
\begin{aligned}
& G_{k, 1}(N, b)=\left.\frac{d^{k}}{d \epsilon^{k}} \mathcal{G}_{1}(N, b, \epsilon)\right|_{\epsilon=0} \\
& G_{k, 2}(N, b)=\left.\frac{d^{k}}{d \epsilon^{k}} \mathcal{G}_{2}(N, b, \epsilon)\right|_{\epsilon=0} .
\end{aligned}
$$

These admit the integral representation

$$
\begin{aligned}
\mathcal{G}_{1}(N, b, \epsilon)= & \int_{0}^{1} d z z^{N-1} \int_{0}^{\frac{(1-z)^{2}}{4 z}} d \xi J_{0}(b M \sqrt{\xi}) \frac{\xi^{-1+\epsilon}}{\sqrt{(1-z)^{2}-4 z \xi}} \\
& -\frac{1}{2 \epsilon^{2}}-\frac{1}{\epsilon} \int_{0}^{1} d z z^{N-1}\left[\frac{1}{1-z}\right]_{+} \\
\mathcal{G}_{2}(N, \epsilon)= & \int_{0}^{\infty} d \xi\left[(\sqrt{1+\xi}-\sqrt{\xi})^{2 N} J_{0}(b M \sqrt{\xi})-1\right] \xi^{-1+\epsilon} .
\end{aligned}
$$

Expanding the Bessel function in powers of its argument

$$
J_{0}(b M \sqrt{\xi})=\sum_{p=0}^{\infty} \frac{(-1)^{p}}{\Gamma^{2}(p+1)}\left(\frac{b^{2} M^{2}}{4}\right)^{p} \xi^{p}
$$

and integrating term by term we get

$$
\begin{aligned}
\mathcal{G}_{1}(N, b, \epsilon)= & \sum_{p=0}^{\infty}\left[\frac{(-1)^{p}}{\Gamma^{2}(p+1)}\left(\frac{b^{2} M^{2}}{4}\right)^{p} \frac{\Gamma(N-p-\epsilon) \Gamma^{2}(p+\epsilon)}{2 \Gamma(N+p+\epsilon)}\right] \\
& -\frac{1}{2 \epsilon^{2}}+\frac{1}{\epsilon}\left(\psi(N)+\gamma_{E}\right) \\
\mathcal{G}_{2}(N, b, \epsilon)= & \sum_{p=0}^{\infty}\left[\frac{(-1)^{p}}{\Gamma^{2}(p+1)}\left(\frac{b^{2} M^{2}}{4}\right)^{p} \frac{N \Gamma(N-p-\epsilon) \Gamma(2(p+\epsilon))}{2^{2 p+2 \epsilon-1} \Gamma(N+1+p+\epsilon)}\right] \\
& -\frac{1}{\epsilon} .
\end{aligned}
$$

We can now take the large- $b$ limit at fixed $\frac{N}{b}$. Because the $\Gamma$ functions do not depend on $b$, this limit can be taken using the asymptotic expansion

$$
\frac{\Gamma(N-p-\epsilon)}{\Gamma(N+p+\epsilon)}=\left(\frac{1}{N^{2}}\right)^{p+\epsilon}\left(1+\mathcal{O}\left(\frac{1}{N}\right)\right) .
$$

By inserting eq. (4.17) into eqs. (4.15), (4.16) and performing the sum on $p$ we obtain

$$
\begin{aligned}
& \mathcal{G}_{1}(N, b, \epsilon)=\frac{1}{2}\left(\frac{1}{N^{2}}\right)^{\epsilon} \Gamma^{2}(\epsilon){ }_{2} F_{1}\left(\epsilon, \epsilon, 1,-\frac{b^{2} M^{2}}{4 N^{2}}\right)-\frac{1}{2 \epsilon^{2}}+\frac{1}{\epsilon}\left(\ln N+\gamma_{E}\right)+\mathcal{O}\left(\frac{1}{b}\right) \\
& \mathcal{G}_{2}(N, b, \epsilon)=2^{1-2 \epsilon}\left(\frac{1}{N^{2}}\right)^{\epsilon} \Gamma(2 \epsilon){ }_{2} F_{1}\left(\epsilon, \frac{1}{2}+\epsilon, 1,-\frac{b^{2} M^{2}}{4 N^{2}}\right)-\frac{1}{\epsilon}+\mathcal{O}\left(\frac{1}{b}\right) .
\end{aligned}
$$

These provide us with the desired expressions of the generating functions at leading order in the $b \rightarrow \infty$ limit, for fixed $\frac{N}{b}$. 
The derivatives of the generating functions eqs. (4.18)-(4.19) could be performed using recent results $[26,27]$ for the expansion of hypergeometric function in powers of $\epsilon$. However, very compact closed-form expressions can be obtained by replacing the generating functions eqs. (4.18)-(4.19) with suitable expressions which only differ by them by subleading terms. Indeed, because powers of $\ln \xi$ are obtained by differentiation with respect to $\epsilon$ according to eq. (4.11), an expression of the generating functions which reproduces transverse momentum resummation up to $\mathrm{N}^{k} \mathrm{LL}$ accuracy can be obtained by expanding the hypergeometric functions in powers of $\epsilon$, and at each order in $\epsilon$ evaluating its large $b$ limit and retaining the $k+1$ highest powers of $\ln b$. Furthermore, because of the prefactor of $N^{-2 \epsilon}$ in eqs. (4.18)-(4.19) an expression of the generating functions which reproduces inclusive threshold momentum resummation up to $\mathrm{N}^{j} \mathrm{LL}$ accuracy can be obtained by letting $b=0$ and then expanding the hypergeometric functions in powers of $\epsilon$ and retaining the first $j$ orders of the expansion. Hence, any function which reproduces these two behaviors of the original generating functions will lead to the same resummed results to the desired accuracy: up to NNLO this requires $k=j=2$.

For $\mathcal{G}_{1}$, we do this by noting that the hypergeometric function ${ }_{2} F_{1}$ has the asymptotic expansion for large $z$

$$
{ }_{2} F_{1}(\epsilon, \epsilon, 1,-z)=\frac{z^{-\epsilon}}{\Gamma(\epsilon) \Gamma(1-\epsilon)}\left(\ln z-\psi(1-\epsilon)-\psi(\epsilon)-2 \gamma_{E}\right)+\mathcal{O}\left(\frac{1}{z}\right),
$$

and the Taylor expansion

$$
{ }_{2} F_{1}(\epsilon, \epsilon, 1,-z)=1+\epsilon^{2} \operatorname{Li}_{2}(-z)+\mathcal{O}\left(\epsilon^{3}\right)
$$

We can easily combine these two behaviours by first, letting $z \rightarrow 1+z$ on the right-hand side of eq. (4.20): this leads to an expression which coincides with eq. (4.20) as $z \rightarrow \infty$ up to $\mathcal{O}\left(\frac{1}{z}\right)$ corrections, but is regular as $z \rightarrow 0$. Next, we expand the result in powers of $\epsilon$ and we match to the expansion eq. (4.21). Namely, we note that

$$
\frac{(1+z)^{-\epsilon}}{\Gamma(\epsilon) \Gamma(1-\epsilon)}\left(\ln (1+z)-\psi(1-\epsilon)-\psi(\epsilon)-2 \gamma_{E}\right)=1-\epsilon^{2}\left[\frac{1}{2} \ln ^{2}(1+z)+\zeta_{2}\right]+\mathcal{O}\left(\epsilon^{3}\right) \text {. }
$$

But

$$
\operatorname{Li}_{2}(-z)+\frac{1}{2} \ln ^{2}(1+z)+\zeta_{2}=\operatorname{Li}_{2}\left(\frac{1}{1+z}\right)-(\ln (1+z)-\ln (z)) \ln (1+z) .
$$

Hence it is enough to add the left-hand side of eq. (4.23) to the right-hand side of eq. (4.20) after having performed in it the $z \rightarrow 1+z$ shift, to get an interpolation of the hypergeometric function which, if substituted in eq. (4.18), leads to the same result to up to subleading power corrections in the small $p_{\mathrm{T}}$ limit and up to $\mathrm{N}^{3} \mathrm{LL}$ corrections in the threshold limit at the integrated level. This can be increased to $\mathrm{N}^{j} \mathrm{LL}$ by including the expansion in powers of $\epsilon$ in eqs. (4.21), (4.22) up to $j-1$-th order. 
The second term on the right-hand side of eq. (4.23) can be dropped, as it is $O(z)$ as $z \rightarrow 0$ and $O\left(\frac{1}{z}\right)$ as $z \rightarrow \infty$, and so we end up with the result

$$
\begin{aligned}
{ }_{2} F_{1}(\epsilon, \epsilon, 1,-z)= & \frac{(1+z)^{-\epsilon}}{\Gamma(1-\epsilon) \Gamma(\epsilon)}\left(\ln (1+z)-2 \gamma_{E}-\psi(1-\epsilon)-\psi(\epsilon)\right) \\
& +\epsilon^{2} \operatorname{Li}_{2}\left(\frac{1}{1+z}\right)+\mathcal{O}(\mathrm{NNNLL})
\end{aligned}
$$

where the order of the correction means that using eq. (4.24) in the expression eq. (4.18) of the generating function $\mathcal{G}_{1}$ leads to resummed expression which preserve the original accuracy in the small $p_{\mathrm{T}}$ limit, and which are NNLL* accurate in the threshold limit upon integration over $p_{\mathrm{T}}$.

For $\mathcal{G}_{2}$ we use the expansion

$$
{ }_{2} F_{1}\left(\epsilon, \frac{1}{2}+\epsilon, 1,-z\right)=\frac{\sqrt{\pi} 2^{-2 \epsilon} z^{-\epsilon}}{\Gamma\left(\frac{1}{2}+\epsilon\right) \Gamma(1-\epsilon)}+\mathcal{O}\left(\frac{1}{z}\right) .
$$

We note furthermore that $\mathcal{G}_{2}$ generates the integrals which enter in the terms proportional to $\mathcal{B}$ in the resummed expression eq. (4.1). These start at NLL, hence, up to NNLL accuracy, it is sufficient to perform the expansion in powers of $\epsilon$ up to first order, rather than second order as in eq. (4.21). Furthermore, we note that the $\mathcal{O}(\epsilon)$ term in this expansion only receives a contribution from the leading-order contribution to $\mathcal{B}$, which vanishes in the threshold limit $N \rightarrow \infty$, see eq. (A.20). It follows that is enough to reproduce the expansion

$$
{ }_{2} F_{1}(\epsilon, \epsilon, 1,-z)=1+\mathcal{O}(\epsilon) .
$$

This is automatically the case is we simply perform the shift $z \rightarrow 1+z$ on the right-hand side of eq. (4.20). We thus end up with the result

$$
{ }_{2} F_{1}\left(\epsilon, \frac{1}{2}+\epsilon, 1,-z\right)=\frac{\sqrt{\pi} 2^{-2 \epsilon}(1+z)^{-\epsilon}}{\Gamma\left(\frac{1}{2}+\epsilon\right) \Gamma(1-\epsilon)}+\mathcal{O}(\mathrm{NNNLL}),
$$

where again the order of the correction means that using this result in eq. (4.19) leads to resummed expression which preserve the original accuracy in the small $p_{\mathrm{T}}$ limit, and which are NNLL* accurate in the threshold limit upon integration over $p_{\mathrm{T}}$.

Inserting the expanded expressions eqs. (4.24), (4.27) into eq. (4.9), (4.10) and performing the derivatives, we obtain, up to NNLL

$$
\begin{aligned}
G_{k, 1}(N, b)= & \frac{(-1)^{k}}{2}\left[-\frac{1}{k+2} \ln ^{k+2} \chi+\frac{\ln \bar{N}^{2}}{k+1} \ln ^{k+1} \chi+\ln ^{k} \bar{N}^{2} \operatorname{Li}_{2}\left(\frac{\bar{N}^{2}}{\chi}\right)\right. \\
& \left.+\mathcal{O}\left(\ln ^{j} \bar{N}^{2} \ln ^{k-1-j} \chi\right)\right] \\
G_{k, 2}(N, b)= & -\frac{(-1)^{k}}{k+1} \ln ^{k+1} \chi+\mathcal{O}\left(\ln ^{k-1} \chi\right)
\end{aligned}
$$


with

$$
\begin{aligned}
\bar{N} & =N e^{\gamma_{E}} \\
\chi & =\bar{N}^{2}+\frac{b^{2} M^{2}}{b_{0}^{2}} \\
b_{0} & =2 e^{-\gamma_{E}} .
\end{aligned}
$$

Using this result, we can cast eq. (4.1) in the familiar form

$$
\begin{aligned}
\frac{d \hat{\sigma}_{i j}^{\mathrm{tr}}}{d \xi_{p}}\left(N, \xi_{p}, \alpha_{s}\left(M^{2}\right), M^{2}\right) & =\sigma_{0} \int_{0}^{\infty} d b \frac{b}{2} J_{0}\left(b M \sqrt{\xi_{p}}\right)\left(\sqrt{1+\xi_{p}}-\sqrt{\xi_{p}}\right)^{-2 N} \\
& \overline{\mathcal{H}}_{i j}\left(N, \alpha_{s}\left(M^{2}\right)\right) \exp \left[\ln \chi g_{1}\left(\lambda_{N}, \lambda_{\chi}\right)+g_{2}\left(\lambda_{N}, \lambda_{\chi}\right)+\alpha g_{3}\left(\lambda_{N}, \lambda_{\chi}\right)\right] \\
& +\mathcal{O}(\mathrm{NNNLL}) .
\end{aligned}
$$

This is our main result to be used for applications. The functions $g_{1}, g_{2}, g_{3}$ resum the LL, NLL, NNLL contributions respectively, and depend on the two large resummation logs

$$
\begin{aligned}
\lambda_{N} & =\alpha_{s}\left(M^{2}\right) \beta_{0} \ln \bar{N}^{2} \\
\lambda_{\chi} & =\alpha_{s}\left(M^{2}\right) \beta_{0} \ln \chi .
\end{aligned}
$$

Note that the function $\chi$ interpolates between $b^{2}$ at large $b$, and $N^{2}$ when $b=0$ :

$$
\begin{aligned}
& \chi=\frac{b^{2} M^{2}}{b_{0}^{2}}\left[1+\mathcal{O}\left(\frac{1}{b^{2}}\right)\right] \\
& \chi=\bar{N}^{2}\left[1+\mathcal{O}\left(\frac{1}{N^{2}}\right)\right],
\end{aligned}
$$

hence, in the former limit, the form of eq. (4.34) naturally matches the standard expression of transverse momentum resummation of ref. [17].

Evaluating the functions $g_{i}$ up to NNLL explicitly we get

$$
\begin{aligned}
g_{1}\left(\lambda_{\chi}, \lambda_{N}\right)= & \frac{A_{g}^{p_{\mathrm{T}},(1)}}{\beta_{0}}\left(\frac{\lambda_{\chi}+\ln \left(1-\lambda_{\chi}\right)}{\lambda_{\chi}}\right)-\frac{A_{g}^{p_{\mathrm{T}},(1)}}{\beta_{0}} \ln \left(1-\lambda_{\chi}\right) \frac{\lambda_{N}}{\lambda_{\chi}} \\
g_{2}\left(\lambda_{\chi}, \lambda_{N}\right)= & \frac{A_{g}^{p_{\mathrm{T}},(1)} \beta_{1}}{\beta_{0}^{3}}\left[\frac{\lambda_{\chi}+\ln \left(1-\lambda_{\chi}\right)}{1-\lambda_{\chi}}+\frac{1}{2} \ln ^{2}\left(1-\lambda_{\chi}\right)\right] \\
& -\frac{A_{g}^{p_{\mathrm{T}},(2)}}{\beta_{0}^{2}}\left[\ln \left(1-\lambda_{\chi}\right)+\frac{\lambda_{\chi}}{1-\lambda_{\chi}}\right]+\frac{\mathcal{B}_{g}^{(1)}(N)}{\beta_{0}} \ln \left(1-\lambda_{\chi}\right) \\
& -\frac{A_{g}^{p_{\mathrm{T}},(1)} \beta_{1} \lambda_{N}}{\beta_{0}^{3}}\left(\frac{\lambda_{\chi}+\ln \left(1-\lambda_{\chi}\right)}{1-\lambda_{\chi}}\right)+\frac{A_{g}^{p_{\mathrm{T}},(2)} \lambda_{N}}{\beta_{0}^{2}} \frac{\lambda_{\chi}}{1-\lambda_{\chi}}
\end{aligned}
$$




$$
\begin{aligned}
g_{3}\left(\lambda_{\chi}, \lambda_{N}\right)= & \frac{A_{g}^{p_{\mathrm{T}},(1)} \beta_{1}^{2}}{2 \beta_{0}^{4}}\left[\frac{\lambda_{\chi}+\ln \left(1-\lambda_{\chi}\right)}{\left(1-\lambda_{\chi}\right)^{2}}\left(\lambda_{\chi}+\left(1-2 \lambda_{\chi}\right) \ln \left(1-\lambda_{\chi}\right)\right)\right] \\
& +\frac{A_{g}^{p_{\mathrm{T}},(1)} \beta_{2}}{\beta_{0}^{3}}\left[\frac{\left(2-3 \lambda_{\chi}\right) \lambda_{\chi}}{2\left(1-\lambda_{\chi}\right)^{2}}+\ln \left(1-\lambda_{\chi}\right)\right] \\
& -\frac{A_{g}^{p_{\mathrm{T}},(2)} \beta_{1}}{\beta_{0}^{3}}\left[\frac{\left(2-3 \lambda_{\chi}\right) \lambda_{\chi}}{2\left(1-\lambda_{\chi}\right)^{2}}+\frac{\left(1-2 \lambda_{\chi}\right) \ln \left(1-\lambda_{\chi}\right)}{\left(1-\lambda_{\chi}\right)^{2}}\right] \\
& +\frac{\mathcal{B}_{g}^{(1)}(N) \beta_{1}}{\beta_{0}} \frac{\lambda_{\chi}+\ln \left(1-\lambda_{\chi}\right)}{1-\lambda_{\chi}}-\frac{A_{g}^{p_{\mathrm{T}},(3)} \lambda_{\chi}^{2}}{2 \beta_{0}^{2}\left(1-\lambda_{\chi}\right)^{2}}-\frac{\mathcal{B}_{g}^{(2)}(N)}{\beta_{0}} \frac{\lambda_{\chi}}{1-\lambda_{\chi}} \\
& +A_{g}^{p_{\mathrm{T}},(1)} \frac{\lambda_{N}}{1-\lambda_{N}} \operatorname{Li}_{2}\left(\frac{\bar{N}^{2}}{\chi}\right)-\frac{A_{g}^{p_{\mathrm{T}},(1)} \beta_{1}^{2} \lambda_{N}}{2 \beta_{0}^{4}} \frac{\lambda_{\chi}^{2}-\ln ^{2}\left(1-\lambda_{\chi}\right)}{\left(1-\lambda_{\chi}\right)^{2}} \\
& -\frac{A_{g}^{p_{\mathrm{T}},(2)} \beta_{1} \lambda_{N}}{2 \beta_{0}^{3}} \frac{\lambda_{\chi}\left(2-\lambda_{\chi}\right)+2 \ln \left(1-\lambda_{\chi}\right)}{\left(1-\lambda_{\chi}\right)^{2}} \\
& +\frac{A_{g}^{p_{\mathrm{T}},(3)}}{2 \beta_{0}^{2}} \frac{\lambda_{N} \lambda_{\chi}\left(2-\lambda_{\chi}\right)}{\left(1-\lambda_{\chi}\right)^{2}}+\frac{A_{g}^{p_{\mathrm{T}},(1)} \beta_{2}}{2 \beta_{0}^{3}} \frac{\lambda_{N} \lambda_{\chi}^{2}}{\left(1-\lambda_{\chi}\right)^{2}}
\end{aligned}
$$

while the hard function is

$$
\overline{\mathcal{H}}_{i j}\left(N, \alpha_{s}\left(M^{2}\right)\right)=\mathcal{H}_{i j}\left(N, \alpha_{s}\left(M^{2}\right)\right)+\delta_{i j=g} A_{g}^{p_{\mathrm{T}},(1)} \operatorname{Li}_{2}\left(\frac{\bar{N}^{2}}{\chi}\right) .
$$

It is then immediate to see that, replacing $\chi$ with its limiting large $b$ form eq. (4.36) and using the explicit expressions of all the coefficients given in appendix A.2 we recover the expression for transverse momentum resummation of ref. [17] (see specifically eqs. (22)-(24) of that reference).

We can now proceed to the nontrivial consistency check of our procedure, namely, that setting $b=0$ we recover threshold resummation at the inclusive level up to NNLL* accuracy. Up to NLL accuracy, inclusive threshold resummation is entirely determined by the cusp anomalous dimension $A_{g}^{\text {th }}$ [explicitly given in eqs. (A.1)-(A.3)], namely, the coefficient of the most singular contribution to the anomalous dimension as $N \rightarrow \infty$, which in the $\overline{\mathrm{MS}}$ scheme is proportional to $\ln N$ [28]. It follows that threshold resummation is reproduced automatically up to this order if the coefficients of the expansion of the function $A_{g}^{\mathrm{pT}}$ eq. (2.32) coincide with those of the cusp anomalous dimension:

$$
\begin{aligned}
& A_{g}^{\mathrm{p}_{\mathrm{T}},(1)}=A_{g}^{\mathrm{th},(1)} \\
& A_{g}^{\mathrm{p}_{\mathrm{T}},(2)}=A_{g}^{\mathrm{th},(2)},
\end{aligned}
$$

which is of course the case. Note that this fact is nontrivial: indeed $A_{g}^{\mathrm{p}_{\mathrm{T}}}$ is implicitly defined by eq. (2.32), which determines order by order the way resummation coefficients are assigned to the functions $A_{g}^{\mathrm{p}^{\mathrm{T}}}$ and $B_{g}^{\mathrm{pT}}$. Our derivation shows that this effectively amounts to defining $A_{g}^{\mathrm{p} T}$ as the function which includes in the Sudakov exponent terms which are enhanced by $\ln N$, i.e., that controls the resummation of terms proportional to $\ln N \ln b$, which, beyond the leading $\log$ level, is not obviously the same as the cusp anomalous dimension. 
And indeed, starting at the NNLL it is not. At this order and beyond, threshold resummation at the inclusive level also receives a contribution from large-angle gluon emission [29], described by an additional function $D_{g}^{\mathrm{p}^{\mathrm{T}}}$ (see eqs. (A.35)-(A.38). Up to NNLL* accuracy we then recover inclusive threshold resummation setting $b=0$ in eq. (4.33) only if the following relations are satisfied:

$$
\begin{aligned}
A_{g}^{\mathrm{p}_{\mathrm{T}},(3)}+\beta_{0} D_{g}^{\mathrm{p}_{\mathrm{T}},(2)} & =A_{g}^{\mathrm{th},(3)} \\
D_{g}^{\mathrm{p}_{\mathrm{T}},(2)}+2 \tilde{B}_{g}^{\mathrm{p}_{\mathrm{T}},(2)}+2 A_{g}^{\mathrm{p}_{\mathrm{T}},(1)} \zeta_{2} \beta_{0} & =D_{g}^{\mathrm{th},(2)} \\
H_{g g}^{\mathrm{p}_{\mathrm{T}},(1)}+A_{g}^{\mathrm{p}_{\mathrm{T}},(1)} \zeta_{2} & =H_{g g}^{\mathrm{th},(1)},
\end{aligned}
$$

where the coefficients on the right-hand side of eqs. (4.44)-(4.46), which determine NNLL threshold resummation at the inclusive level, are explicitly listed in appendix A.3. It is straightforward to check that eqs. (4.42)-(4.46) are indeed satisfied. The fact that at NNLL and beyond $A_{g}^{\mathrm{pT}}$ does not coincide with the cusp anomalous dimension was first shown using SCET arguments in refs. [18, 30], where it was derived from the breaking of a symmetry of the classical SCET Lagrangian called "collinear anomaly". We now see that this simply means that the coefficient of $\ln N$ in the anomalous dimension, and the coefficient of $\ln N \ln b$ in transverse momentum resummation, do not coincide, because the latter receives contribution from interference between soft virtual corrections, controlled by $D_{g}^{p_{\mathrm{T}}}$, and collinear emission.

Note that the accuracy of our results is NNLL*, rather than NNLL, because $H_{g g}^{\mathrm{p}_{\mathrm{T}},(2)}$ differs from $H_{g g}^{\mathrm{th},(2)}$ by terms proportional to $g_{4}$, which we do not fully include. All results presented here apply to Higgs production in gluon fusion; however, results for Drell-Yan production have the same structure and are obtained by simply replacing the expressions of the functions $A_{g}^{p_{\mathrm{T}}}$ and $\mathcal{B}_{g}(N)$ with their quark counterparts $A_{q}^{p_{\mathrm{T}}}$ and $\mathcal{B}_{q}(N)$.

\section{Conclusions}

In this paper we have constructed an expression for the transverse momentum distribution of a colorless object in perturbative QCD which reduces to transverse momentum resummation in the small $p_{\mathrm{T}}$ limit at fixed $x$ and to threshold resummation in the $x \rightarrow 1$ limit at fixed $p_{\mathrm{T}}$, and which, furthermore, gives threshold resummation at the inclusive level when integrated over transverse momentum. Our combined resummed expression is the matched formula eq. (4.7) with eqs. (4.5)-(4.6). Its main original ingredient is the modified transverse momentum resummation formula, given as a master integral formula in eq. (4.1) and as a compact closed-form expression in eq. (4.33). In fact, eq. (4.33) is our main new result: it provides a modified expression for transverse momentum resummation which automatically reproduces threshold resummation upon integration over $p_{T}$.

The interest in this construction is threefold. First, we provide resummed expressions which can be used for phenomenology at a differential level, allowing for an improvement of the transverse momentum distribution through threshold resummation in a way that holds for all values of $p_{\mathrm{T}}$ and which matches onto inclusive results which have been similarly improved. Second, our results, when expanded out to finite order in $\alpha_{s}$ provide powerful constraints on higher-order perturbative corrections, which may be used as consistency check of 
full calculations, and as a means of constructing approximate results for yet unknown higher order terms. Finally, we elucidate the relation between the collinear and soft logs which drive the transverse momentum distribution and the total cross section in the soft limit.

The virtues of our final result can be perhaps best understood by comparing it to other related results which have been previously derived. In ref. [31] a joint resummation for Higgs production up to NLL was derived, by studying singular eikonal emission within the web formalism. A resummed result was obtained in terms of an interpolating function $\bar{\chi}=\frac{b M}{b_{0}}+\bar{N}$ which can be compared to our resummation $\log \lambda_{\chi}$ eq. (4.35) with $\chi$ eq. (4.31). This result was recently extended up to NNLL in ref. [6] by means of a suitable Ansatz. We have checked that our result reproduces that of these references in the sense that our $g_{1}$ and $g_{2}$ resummation functions eqs. (4.38), (4.39) coincide with the corresponding expressions of ref. [31] and $g_{3}$ ref. [6] once differences in notation are accounted for. However, the logs of the interpolating function $\bar{\chi}$ of ref. [31] produce terms which are subleading in the small $p_{\mathrm{T}}$ limit but induce in the result of ref. [31] unphysical $\operatorname{logs}$ of $\frac{N}{b}$ which are not present at any finite order. In order to remove them, a phenomenological modification of $\chi$ was proposed in ref. [5] and used in ref. [31], $\bar{\chi}_{\text {phen }}(\eta)=\frac{b M}{b_{0}}+\frac{\bar{N}}{1+\eta \frac{b M}{2 N}}$, dependent on a free parameter $\eta$ and such that if $\eta \neq 0$ the spurious large $N$ behaviour is removed up to order $b^{-1}$. Our result avoids these ad-hoc manipulations. Finally, in ref. [8], a NNLL joint resummation in SCET was performed. This resummation is directly performed in $x$ and $p_{\mathrm{T}}$ space, and it does not appear to reproduce threshold resummation at the inclusive level upon integration over transverse momentum.

Besides phenomenological studies, future directions of progress include the possibility of merging the result of this paper with the recent high energy resummation for transverse momentum distributions performed at fixed $-p_{\mathrm{T}}$ in refs. [32, 33], and already matched to transverse momentum resummation in ref. [34], with the eventual goal of deriving resummation of the fully exclusive cross section in all kinematic limits.

\section{Acknowledgments}

We are grateful to Fabrizio Caola for a critical reading of the manuscript and several interesting observations. We also thank Giancarlo Ferrera and Simone Marzani for useful discussions.

\section{A Explicit expressions}

\section{A.1 Threshold resummation at fixed $p_{\mathrm{T}}$}

We give here explicit expressions of the coefficients which determine threshold resummation for Higgs production in gluon fusion in the pointlike limit up to NNLL* accuracy when used in the expression eq. (2.26) of $\frac{d \hat{\sigma}_{i j}^{\text {th }}}{d \xi_{p}}$. The cusp anomalous dimensions $A_{g}^{\text {th }}\left(\alpha_{s}\right)$ and $A_{q}^{\text {th }}\left(\alpha_{s}\right)$ i.e. the contribution to the $P_{g g}$ and $P_{q q}$ splitting function respectively which are 
proportional to a plus distribution are given by (see e.g. ref. [14]):

$$
\begin{aligned}
A_{c}^{\text {th }}\left(\alpha_{s}\right)= & A_{c}^{\text {th },(1)}\left(\frac{\alpha_{s}}{\pi}\right)+A_{c}^{\text {th },(2)}\left(\frac{\alpha_{s}}{\pi}\right)^{2}+A_{c}^{\text {th },(3)}\left(\frac{\alpha_{s}}{\pi}\right)^{3}+\mathcal{O}\left(\alpha_{s}^{4}\right) \\
A_{c}^{\text {th },(1)}= & C_{c} \\
A_{c}^{\text {th },(2)}= & \frac{C_{c}}{2}\left[C_{\mathrm{A}}\left(\frac{67}{18}-\zeta_{2}\right)-\frac{5}{9} n_{f}\right] \\
A_{c}^{\text {th },(3)}= & C_{c}\left[\left(\frac{245}{96}-\frac{67}{36} \zeta_{2}+\frac{11}{8} \zeta_{4}+\frac{11}{24} \zeta_{3}\right) C_{\mathrm{A}}^{2}+\left(-\frac{209}{432}+\frac{5}{18} \zeta_{2}-\frac{7}{12} \zeta_{3}\right) C_{\mathrm{A}} n_{f}\right. \\
& \left.+\left(-\frac{55}{96}+\frac{1}{2} \zeta_{3}\right) C_{\mathrm{F}} n_{f}-\frac{1}{108} n_{f}^{2}\right]
\end{aligned}
$$

with $C_{c}=C_{\mathrm{A}}$ if $c=g$ is a gluon and $C_{c}=C_{\mathrm{F}}$ if $c=q$. Furthermore

$$
\begin{aligned}
B_{c}^{\mathrm{th}}\left(\alpha_{s}\right)= & B_{c}^{\mathrm{th},(1)}\left(\frac{\alpha_{s}}{\pi}\right)+B_{c}^{\mathrm{th},(2)}\left(\frac{\alpha_{s}}{\pi}\right)^{2}+\mathcal{O}\left(\alpha_{s}^{3}\right) \\
B_{q}^{\mathrm{th},(1)}=- & \frac{3}{4} C_{\mathrm{F}} \\
B_{q}^{\mathrm{th},(2)}= & \frac{1}{16}\left[C_{\mathrm{F}}^{2}\left(-\frac{3}{2}+12 \zeta_{2}-24 \zeta_{3}\right)+C_{\mathrm{F}} C_{\mathrm{A}}\left(-\frac{3155}{54}+\frac{44}{3} \zeta_{2}+40 \zeta_{3}\right)\right. \\
& \left.+C_{\mathrm{F}} n_{f}\left(\frac{247}{27}-\frac{8}{3} \zeta_{2}\right)\right] \\
B_{g}^{\mathrm{th},(1)}= & -\beta_{0}=-\frac{11}{12} C_{\mathrm{A}}+\frac{1}{6} n_{f}, \\
B_{g}^{\mathrm{th},(2)}= & \frac{1}{16}\left[C_{\mathrm{A}}^{2}\left(-\frac{611}{9}+\frac{88}{3} \zeta_{2}+16 \zeta_{3}\right)+C_{\mathrm{A}} n_{f}\left(\frac{428}{27}-\frac{16}{3} \zeta_{2}\right)+2 C_{\mathrm{F}} n_{f}-\frac{20}{27} n_{f}^{2}\right] .
\end{aligned}
$$

Note that both quark and gluon channel expressions are necessary for NNLL* accuracy.

We now turn to the LO coefficient functions $C_{0}\left(N, \xi_{p}\right)$ in eq. (2.26): after factoring the leading-order total cross section

$$
\sigma_{0}=\frac{\alpha_{s}^{2} \sqrt{2} G_{\mathrm{F}}}{576 \pi}
$$

they are given by

$$
\begin{aligned}
& \frac{d \hat{\sigma}_{g g \rightarrow g H}^{\mathrm{LO}}}{d \xi_{p}}\left(N, \xi_{p}\right)=\frac{2 \alpha_{s} C_{\mathrm{A}}}{\pi} \frac{1}{\xi_{p}} \frac{\Gamma\left(\frac{1}{2}\right) \Gamma(N)}{\Gamma\left(N+\frac{1}{2}\right)}\left({ }_{2} F_{1}\left(\frac{1}{2}, N, N+\frac{1}{2},\left(\sqrt{1+\xi_{p}}-\sqrt{\xi_{p}}\right)^{4}\right)\right. \\
& -2 \frac{1+\xi_{p}}{\left(\sqrt{1+\xi_{p}}+\sqrt{\xi_{p}}\right)^{2}} \frac{N}{N+\frac{1}{2}}{ }_{2} F_{1}\left(\frac{1}{2}, N+1, N+\frac{3}{2},\left(\sqrt{1+\xi_{p}}-\sqrt{\xi_{p}}\right)^{4}\right) \\
& +\frac{\left(1+\xi_{p}\right)\left(3+\xi_{p}\right)}{\left(\sqrt{1+\xi_{p}}+\sqrt{\xi_{p}}\right)^{4}} \frac{N(N+1)}{\left(N+\frac{1}{2}\right)\left(N+\frac{3}{2}\right)}{ }_{2} F_{1}\left(\frac{1}{2}, N+2, N+\frac{5}{2},\left(\sqrt{1+\xi_{p}}-\sqrt{\xi_{p}}\right)^{4}\right) \\
& -2 \frac{1+\xi_{p}}{\left(\sqrt{1+\xi_{p}}+\sqrt{\xi_{p}}\right)^{6}} \frac{N(N+1)(N+2)}{\left(N+\frac{1}{2}\right)\left(N+\frac{3}{2}\right)\left(N+\frac{5}{2}\right)}{ }_{2} F_{1}\left(\frac{1}{2}, N+3, N+\frac{7}{2},\left(\sqrt{1+\xi_{p}}-\sqrt{\xi_{p}}\right)^{4}\right)
\end{aligned}
$$




$$
\begin{aligned}
& +\frac{1}{\left(\sqrt{1+\xi_{p}}+\sqrt{\xi_{p}}\right)^{8}} \frac{N(N+1)(N+2)(N+3)}{\left(N+\frac{1}{2}\right)\left(N+\frac{3}{2}\right)\left(N+\frac{5}{2}\right)\left(N+\frac{7}{2}\right)} \\
& \left.{ }_{2} F_{1}\left(\frac{1}{2}, N+4, N+\frac{9}{2},\left(\sqrt{1+\xi_{p}}-\sqrt{\xi_{p}}\right)^{4}\right)\right) \\
& \frac{d \hat{\sigma}_{g q \rightarrow q H}^{\mathrm{LO}}}{d \xi_{p}}\left(N, \xi_{p}\right)=\frac{\alpha_{s} C_{\mathrm{F}}}{\pi} \frac{1}{\xi_{p}} \frac{\Gamma\left(\frac{1}{2}\right) \Gamma(N)}{\Gamma\left(N+\frac{1}{2}\right)}\left({ }_{2} F_{1}\left(\frac{1}{2}, N, N+\frac{1}{2},\left(\sqrt{1+\xi_{p}}-\sqrt{\xi_{p}}\right)^{4}\right)\right. \\
& -\frac{\left(4+3 \xi_{p}\right)}{\left(\sqrt{1+\xi_{p}}+\sqrt{\xi_{p}}\right)^{2}} \frac{N}{N+\frac{1}{2}}{ }_{2} F_{1}\left(\frac{1}{2}, N+1, N+\frac{3}{2},\left(\sqrt{1+\xi_{p}}-\sqrt{\xi_{p}}\right)^{4}\right) \\
& +3 \frac{1+\xi_{p}}{\left(\sqrt{1+\xi_{p}}+\sqrt{\xi_{p}}\right)^{4}} \frac{N(N+1)}{\left(N+\frac{1}{2}\right)\left(N+\frac{3}{2}\right)}{ }_{2} F_{1}\left(\frac{1}{2}, N+2, N+\frac{5}{2},\left(\sqrt{1+\xi_{p}}-\sqrt{\xi_{p}}\right)^{4}\right) \\
& \left.-\frac{1}{\left(\sqrt{1+\xi_{p}}+\sqrt{\xi_{p}}\right)^{6}} \frac{N(N+1)(N+2)}{\left(N+\frac{1}{2}\right)\left(N+\frac{3}{2}\right)\left(N+\frac{5}{2}\right)}{ }_{2} F_{1}\left(\frac{1}{2}, N+3, N+\frac{7}{2},\left(\sqrt{1+\xi_{p}}-\sqrt{\xi_{p}}\right)^{4}\right)\right) \\
& \frac{d \hat{\sigma}_{q q \rightarrow g H}^{\mathrm{LO}}}{d \xi_{p}}\left(N, \xi_{p}\right)=\frac{2 \alpha_{s} C_{\mathrm{F}}^{2}}{\pi} \frac{1}{\left(\sqrt{1+\xi_{p}}+\sqrt{\xi_{p}}\right)^{2}}\left({ }_{2} F_{1}\left(\frac{1}{2}, N, N+\frac{1}{2},\left(\sqrt{1+\xi_{p}}-\sqrt{\xi_{p}}\right)^{4}\right)\right. \\
& -2 \frac{\left(1+\xi_{p}\right)}{\left(\sqrt{1+\xi_{p}}+\sqrt{\xi_{p}}\right)^{2}} \frac{N}{N+\frac{1}{2}}{ }_{2} F_{1}\left(\frac{1}{2}, N+1, N+\frac{3}{2},\left(\sqrt{1+\xi_{p}}-\sqrt{\xi_{p}}\right)^{4}\right) \\
& \left.+\frac{1}{\left(\sqrt{1+\xi_{p}}+\sqrt{\xi_{p}}\right)^{4}} \frac{N(N+1)}{\left(N+\frac{1}{2}\right)\left(N+\frac{3}{2}\right)}{ }_{2} F_{1}\left(\frac{1}{2}, N+2, N+\frac{5}{2},\left(\sqrt{1+\xi_{p}}-\sqrt{\xi_{p}}\right)^{4}\right)\right),
\end{aligned}
$$

where ${ }_{2} F_{1}$ is the Hypergeometric Function.

Finally, the matching constant

$$
g_{0 i j}\left(\xi_{p}\right)=1+g_{0}^{(1)}{ }_{i j}\left(\xi_{p}\right)\left(\frac{\alpha_{s}}{\pi}\right)+\mathcal{O}\left(\alpha_{s}^{2}\right)
$$

is given in ref. [4]. We have recomputed it independently, obtaining

$$
\begin{aligned}
& g_{0}^{(1)} g_{g}\left(\xi_{p}\right)=\frac{67}{36} C_{\mathrm{A}}-\frac{5}{18} n_{f}+C_{\mathrm{A}} \zeta_{2}-\beta_{0} \ln \frac{\xi_{p}}{1+\xi_{p}}-\frac{1}{8} C_{\mathrm{A}} \ln ^{2} \frac{\xi_{p}}{1+\xi_{p}} \\
& \quad+2 C_{\mathrm{A}} \operatorname{Li}_{2}\left(1-\frac{\sqrt{\xi_{p}}}{\sqrt{1+\xi_{p}}}\right)+C_{\mathrm{A}} \ln \left(1-\frac{\sqrt{\xi_{p}}}{\sqrt{1+\xi_{p}}}\right) \ln \frac{\xi_{p}}{1+\xi_{p}} \\
& \quad-\frac{1}{2} C_{\mathrm{A}} \ln \left(1+\frac{\sqrt{\xi_{p}}}{\sqrt{1+\xi_{p}}}\right) \ln \frac{\xi_{p}}{1+\xi_{p}}+\frac{1}{2} C_{\mathrm{A}} \ln ^{2}\left(1+\frac{\sqrt{\xi_{p}}}{\sqrt{1+\xi_{p}}}\right)+2 \beta_{0} \ln ^{2}\left(1+\frac{\sqrt{\xi_{p}}}{\sqrt{1+\xi_{p}}}\right) \\
& \quad+C_{\mathrm{A}} \operatorname{Li}_{2}\left(\frac{2 \sqrt{\xi_{p}}}{\sqrt{1+\xi_{p}}+\sqrt{\xi_{p}}}\right)-\frac{\left(C_{\mathrm{A}}-n_{f}\right)\left(\sqrt{\xi_{p}} \sqrt{1+\xi_{p}}\left(1+\xi_{p}\right)-2 \xi_{p}-\xi_{p}^{2}\right)}{6\left(1+8 \xi_{p}+9 \xi_{p}^{2}\right)} \\
& g_{0}^{(1)}{ }_{g q}\left(\xi_{p}\right)=-\frac{7}{4} C_{\mathrm{F}}+\frac{134}{36} C_{\mathrm{A}}-\frac{20}{36} n_{f}-8 C_{\mathrm{F}} \zeta_{2}+12 C_{\mathrm{A}} \zeta_{2}-4 \beta_{0} \ln \frac{\xi_{p}}{1+\xi_{p}}+\frac{3}{2} C_{\mathrm{F}} \ln \frac{\xi_{p}}{1+\xi_{p}} \\
& \quad-\frac{1}{2} C_{\mathrm{A}} \ln ^{2} \frac{\xi_{p}}{1+\xi_{p}}+4\left(C_{\mathrm{F}}+C_{\mathrm{A}}\right) \operatorname{Li}_{2}\left(2,1-\frac{\sqrt{\xi_{p}}}{\sqrt{1+\xi_{p}}}\right)
\end{aligned}
$$




$$
\begin{aligned}
& +\frac{2\left(C_{\mathrm{A}}-C_{\mathrm{F}}\right)\left(1+3 \xi_{p}+3 \sqrt{\xi_{p}} \sqrt{1+\xi_{p}}\right)}{2 \sqrt{\xi_{p}} \sqrt{1+\xi_{p}}+1+3 \xi_{p}}+8 \beta_{0} \ln \left(1+\frac{\sqrt{\xi_{p}}}{\sqrt{1+\xi_{p}}}\right) \\
& -3 C_{\mathrm{F}} \ln \left(1+\frac{\sqrt{\xi_{p}}}{\sqrt{1+\xi_{p}}}\right)+2 C_{\mathrm{F}} \ln \left(1-\frac{\sqrt{\xi_{p}}}{\sqrt{1+\xi_{p}}}\right) \ln \frac{\xi_{p}}{1+\xi_{p}} \\
& +2 C_{\mathrm{A}} \ln \left(1-\frac{\sqrt{\xi_{p}}}{\sqrt{1+\xi_{p}}}\right) \ln \frac{\xi_{p}}{1+\xi_{p}}-2 C_{\mathrm{F}} \ln \left(1+\frac{\sqrt{\xi_{p}}}{\sqrt{1+\xi_{p}}}\right) \ln \frac{\xi_{p}}{1+\xi_{p}} \\
& -2 C_{\mathrm{F}} \ln ^{2}\left(1+\frac{\sqrt{\xi_{p}}}{\sqrt{1+\xi_{p}}}\right)+4 C_{\mathrm{F}} \operatorname{Li}_{2}\left(\frac{2 \sqrt{\xi_{p}}}{\sqrt{1+\xi_{p}}+\sqrt{\xi_{p}}}\right) \\
& g_{0}^{(1)}{ }_{q q}\left(\xi_{p}\right)=-\frac{9}{2} C_{\mathrm{F}}+\frac{79}{12} C_{\mathrm{A}}-\frac{5}{6} n_{f}+12 C_{\mathrm{F}} \zeta_{2}-10 C_{\mathrm{A}} \zeta_{2}-\frac{\left(C_{\mathrm{F}}-C_{\mathrm{A}}\right) \sqrt{1+\xi_{p}}}{\sqrt{\xi_{p}}} \\
& +4 C_{\mathrm{F}} \operatorname{Li}_{2}\left(1-\frac{\sqrt{\xi_{p}}}{\sqrt{1+\xi_{p}}}\right)-\frac{3}{4} C_{\mathrm{F}} \ln \frac{\xi_{p}}{1+\xi_{p}}-\beta_{0} \ln \frac{\xi_{p}}{1+\xi_{p}}+\frac{1}{4} C_{\mathrm{A}} \ln ^{2} \frac{\xi_{p}}{1+\xi_{p}} \\
& -\frac{1}{2} C_{\mathrm{F}} \ln ^{2} \frac{\xi_{p}}{1+\xi_{p}}+2 C_{\mathrm{F}} \ln \left(1-\frac{\xi_{p}}{\sqrt{1+\xi_{p}}}\right) \ln \frac{3}{1+\xi_{p}}+\frac{3}{2} C_{\mathrm{F}} \ln \left(1+\frac{\sqrt{\xi_{p}}}{\sqrt{1+\xi_{p}}}\right) \\
& +2 \beta_{0} \ln \left(1+\frac{\sqrt{\xi_{p}}}{\sqrt{1+\xi_{p}}}\right)+C_{\mathrm{A}} \ln ^{2}\left(1+\frac{\sqrt{\xi_{p}}}{\sqrt{1+\xi_{p}}}\right) \\
& -C_{\mathrm{A}} \ln \left(1+\frac{\xi_{p}}{\sqrt{1+\xi_{p}}}\right) \ln \frac{\xi_{p}}{1+\xi_{p}}+2 C_{\mathrm{A}} \operatorname{Li}_{2}\left(\frac{2 \sqrt{\xi_{p}}}{\sqrt{1+\xi_{p}}+\sqrt{\xi_{p}}}\right) .
\end{aligned}
$$

\section{A.2 Transverse momentum resummation}

We collect expressions of the coefficients which determine our generalized transverse momentum resummation $\frac{d \hat{\sigma}_{i j}^{\mathrm{tr}^{\prime}}}{d \xi_{p}}$ eq. (4.1) for Higgs production in gluon fusion up to NNLL order. They are based on the results of refs. [16, 17, 19, 25, 35, 36]) for the standard transverse momentum resummation $\frac{d \hat{\sigma}_{j}^{\text {tr }}}{d \xi_{p}}$ eq. (2.31). As shown in those references, the functions $A^{p_{\mathrm{T}}}$ and $B^{p_{\mathrm{T}}}$ in eq. (2.32) depend on the leading-order partonic subprocess; they will thus be labeled by a subscript $g$ to denote the gluon channel.

The function $A_{g}^{p_{\mathrm{T}}}$ in eq. (4.1) is a series of constants:

$$
A_{g}^{p_{\mathrm{T}}}\left(\alpha_{s}\right)=A_{g}^{p_{\mathrm{T}},(1)}\left(\frac{\alpha_{s}}{\pi}\right)+A_{g}^{p_{\mathrm{T}},(2)}\left(\frac{\alpha_{s}}{\pi}\right)^{2}+A_{g}^{p_{\mathrm{T}},(3)}\left(\frac{\alpha_{s}}{\pi}\right)^{3}+\mathcal{O}\left(\alpha_{s}^{4}\right),
$$

where

$$
\begin{aligned}
A_{g}^{p_{\mathrm{T}},(1)}= & C_{\mathrm{A}} \\
A_{g}^{p_{\mathrm{T}},(2)}= & \frac{C_{\mathrm{A}}}{2}\left[C_{\mathrm{A}}\left(\frac{67}{18}-\zeta_{2}\right)-\frac{5}{9} n_{f}\right] \\
A_{g}^{\mathrm{p}_{\mathrm{T}},(3)}= & \frac{C_{\mathrm{A}}}{4}\left[C_{\mathrm{A}}^{2}\left(\frac{15503}{648}-\frac{67}{9} \zeta_{2}-11 \zeta_{3}+\frac{11}{2} \zeta_{4}\right)+C_{\mathrm{F}} n_{f}\left(-\frac{55}{24}+2 \zeta_{3}\right)\right. \\
& \left.+C_{\mathrm{A}} n_{f}\left(-\frac{2051}{324}+\frac{10}{9} \zeta_{2}\right)-\frac{25}{81} n_{f}^{2}\right] .
\end{aligned}
$$


The function $\mathcal{B}_{g}(N)$, is a two by two matrix, since PDFs evolution only involves the singlet sector in the case of Higgs production. It includes all exponentiated terms which vanish as $N \rightarrow \infty$ and admits the following expansion in $\alpha_{s}$

$$
\begin{aligned}
\mathcal{B}_{g}\left(N, \alpha_{s}\right) & =\mathcal{B}_{g}^{(1)}(N)\left(\frac{\alpha_{s}}{\pi}\right)+\mathcal{B}_{g}^{(2)}(N)\left(\frac{\alpha_{s}}{\pi}\right)^{2}+\mathcal{O}\left(\alpha_{s}^{3}\right), \\
\mathcal{B}_{g}^{(1)}(N) & =2 \gamma^{\mathrm{reg},(1)}(N) \\
\mathcal{B}_{g}^{(2)}(N) & =\tilde{B}^{\mathrm{p}_{\mathrm{T}},(2)}+2 \gamma^{\mathrm{reg},(2)}(N),
\end{aligned}
$$

where both $\gamma^{\text {reg,(i) }}$ and $\tilde{B}^{\mathrm{p}_{\mathrm{T}},(2)}$ are two by two matrices defined as

$$
\begin{array}{rlrl}
\gamma_{j k}^{\text {reg,(i) }}(N) & =\gamma_{j k}^{(i)}(N) & \text { if } j \neq k \neq g \\
\gamma_{g g}^{\text {reg,(i) }}(N) & =\gamma_{g g}^{(i)}(N)+A_{g}^{\text {th, (i) }}\left(\ln N+\gamma_{E}\right)-\delta P_{g g}^{i} & \\
\tilde{B}_{j k}^{\mathrm{p}_{\mathrm{T}},(2)} & =0 & & \text { if } j \neq k \neq g \\
\tilde{B}_{g g}^{\mathrm{p}_{\mathrm{T}},(2)} & =\beta_{0} C_{\mathrm{A}} \zeta_{2} &
\end{array}
$$

with $C_{\mathrm{A}}=3, C_{\mathrm{F}}=\frac{4}{3}$. Here, $\gamma^{(i)}$ are the coefficients of $\left(\frac{\alpha_{s}}{\pi}\right)^{i}$ in the expansions of the Altarelli-Parisi anomalous dimensions, the cusp anomalous dimension $A^{\text {th,(i) }}$ was given in eqs. (A.1)-(A.3) and $\delta P_{g g}^{(i)}$ is the coefficient of $\left(\frac{\alpha_{s}}{\pi}\right)^{i} \delta(1-z)$ in the expansion of the splitting function $P_{g g}(z)$. From eqs. (A.22)-(A.23), it is easy to see that

$$
\gamma^{\mathrm{reg},(\mathrm{i})}(N)=\mathcal{O}\left(\frac{1}{N}\right)
$$

at large $N$.

The function $\mathcal{H}_{i j}\left(N, \alpha_{s}\left(M^{2}\right)\right)$ is given in refs. [17, 25], and it is defined factoring out the inclusive cross section eq. (A.8). The coefficients which control logarithmically enhanced contributions as $N \rightarrow \infty$ are

$$
\begin{aligned}
D_{g}^{p_{\mathrm{T}}}\left(\alpha_{s}\right) & =D_{g}^{p_{\mathrm{T}},(1)}\left(\frac{\alpha_{s}}{\pi}\right)+D_{g}^{p_{\mathrm{T}},(2)}\left(\frac{\alpha_{s}}{\pi}\right)^{2}+\mathcal{O}\left(\alpha_{s}^{3}\right) \\
D_{g}^{p_{\mathrm{T}},(1)} & =0 \\
D_{g}^{p_{\mathrm{T}},(2)} & =C_{\mathrm{A}}^{2}\left(-\frac{101}{27}+\frac{7}{2} \zeta_{3}\right)+\frac{14}{27} C_{\mathrm{A}} n_{f}
\end{aligned}
$$

while the term $\mathcal{H}_{i j}^{0}\left(\alpha_{s}\right)$ (constant as $N \rightarrow \infty$ ) is

$$
\begin{aligned}
\mathcal{H}_{i j}^{0}\left(\alpha_{s}\right) & =0 \quad \text { if } i \neq j \neq g \\
\mathcal{H}_{g g}^{0}\left(\alpha_{s}\right) & =1+\mathcal{H}_{g g}^{0,(1)}\left(\frac{\alpha_{s}}{\pi}\right)+\mathcal{H}_{g g}^{0,(2)}\left(\frac{\alpha_{s}}{\pi}\right)^{2}+\mathcal{O}\left(\alpha_{s}^{3}\right) \\
\mathcal{H}_{g g}^{0,(1)} & =3 C_{\mathrm{A}} \zeta_{2}, \\
\mathcal{H}_{g g}^{0,(2)} & =C_{\mathrm{A}}^{2}\left(\frac{93}{16}+\frac{67}{12} \zeta_{2}-\frac{55}{18} \zeta_{3}+\frac{65}{8} \zeta_{4}\right)+C_{\mathrm{A}} n_{f}\left(-\frac{5}{3}-\frac{5}{6} \zeta_{2}-\frac{4}{9} \zeta_{3}\right) .
\end{aligned}
$$


Finally, terms which vanish as $N \rightarrow \infty$ are [17, 25]

$$
\begin{aligned}
& \mathcal{H}_{g g}^{\text {f.o., }(1)}(N)=0 \\
& \mathcal{H}_{g q}^{\text {f.o., }(1)}(N)=\mathcal{H}_{q g}^{(1)}(N)=\frac{1}{2} C_{\mathrm{F}} \frac{1}{N+1} .
\end{aligned}
$$

The complicated $\mathcal{O}\left(\alpha_{s}^{2}\right)$ coefficients can be obtained by Mellin transform of coefficients given in ref. [25]:

$$
\begin{aligned}
& \mathcal{H}_{i j}^{\text {f.o, }(2)}(N)=\int_{0}^{1} d z z^{N-1} \mathcal{H}_{g g \leftarrow i j}^{H(2)}(z), \quad i j=q q, q g \\
& \mathcal{H}_{g g}^{\text {f.o, }(2)}(N)=-\mathcal{H}_{g g}^{0,(2)}+D_{g}^{p_{\mathrm{T}},(2)} \ln N+\int_{0}^{1} d z z^{N-1} \mathcal{H}_{g g \leftarrow g g}^{H(2)}(z),
\end{aligned}
$$

with $\mathcal{H}_{g g \leftarrow i j}^{H(2)}$ given in eqs. (22-24) of ref. [25].

\section{A.3 Threshold resummation for inclusive cross section}

We provide here the remaining coefficients which fully determine NNLL* threshold resummation at the inclusive level [14, 29]. This can written as

$$
\hat{\sigma}^{\mathrm{res}}(N)=\sigma_{0} H_{g g}^{\mathrm{th}} \exp [G(N)]
$$

with

$$
G(N)=\int_{0}^{1} d z \frac{z^{N-1}-1}{1-z}\left[2 \int_{M^{2}}^{(1-z)^{2} M^{2}} \frac{d q^{2}}{q^{2}} A_{g}^{\text {th }}\left(\alpha_{s}\left(q^{2}\right)\right)+D_{g}^{\text {th }}\left(\alpha_{s}\left((1-z)^{2} M^{2}\right)\right)\right] .
$$

The the cusp anomalous dimension $A^{\text {th,(i) }}$ was given in eqs. (A.1)-(A.3). The function $D_{g}\left(\alpha_{s}\left((1-z)^{2} M^{2}\right)\right)$, which contains contributions from large-angle (non collinear) soft gluon emission, is given by

$$
\begin{aligned}
D_{g}^{\mathrm{th}}\left(\alpha_{s}\right) & =D_{g}^{\mathrm{th},(1)}\left(\frac{\alpha_{s}}{\pi}\right)+D_{g}^{\mathrm{th},(2)}\left(\frac{\alpha_{s}}{\pi}\right)^{2}+\mathcal{O}\left(\alpha_{s}^{3}\right) \\
D_{g}^{\mathrm{th},(1)} & =0 \\
D_{g}^{\mathrm{th},(2)} & =C_{\mathrm{A}}\left(C_{\mathrm{A}}\left(-\frac{101}{27}+\frac{11}{3} \zeta_{2}+\frac{7}{2} \zeta_{3}\right)+n_{f}\left(\frac{14}{27}-\frac{2}{3} \zeta_{2}\right)\right)
\end{aligned}
$$

and the hard function is

$$
\begin{aligned}
H_{g g}^{\mathrm{th}}(a s) & =1+H_{g g}^{\mathrm{th},(1)}\left(\frac{\alpha_{s}}{\pi}\right)+\mathcal{O}\left(\alpha_{s}^{2}\right) \\
H_{g g}^{\mathrm{th},(1)} & =4 C_{\mathrm{A}} \zeta_{2} .
\end{aligned}
$$


Open Access. This article is distributed under the terms of the Creative Commons Attribution License (CC-BY 4.0), which permits any use, distribution and reproduction in any medium, provided the original author(s) and source are credited.

\section{References}

[1] J.C. Collins, D.E. Soper and G.F. Sterman, Transverse Momentum Distribution in Drell-Yan Pair and $W$ and $Z$ Boson Production, Nucl. Phys. B 250 (1985) 199 [inSPIRE].

[2] S. Catani and L. Trentadue, Resummation of the QCD Perturbative Series for Hard Processes, Nucl. Phys. B 327 (1989) 323 [INSPIRE].

[3] G.F. Sterman, Summation of Large Corrections to Short Distance Hadronic Cross-Sections, Nucl. Phys. B 281 (1987) 310 [inSPIRE].

[4] D. de Florian, A. Kulesza and W. Vogelsang, Threshold resummation for high-transverse-momentum Higgs production at the LHC, JHEP 02 (2006) 047 [hep-ph/0511205] [INSPIRE].

[5] E. Laenen, G.F. Sterman and W. Vogelsang, Recoil and threshold corrections in short distance cross-sections, Phys. Rev. D 63 (2001) 114018 [hep-ph/0010080] [INSPIRE].

[6] S. Marzani and V. Theeuwes, Vector boson production in joint resummation, JHEP 02 (2017) 127 [arXiv:1612.01432] [INSPIRE].

[7] Y. Li, D. Neill and H.X. Zhu, An Exponential Regulator for Rapidity Divergences, submitted to Phys. Rev. D (2016) [arXiv:1604.00392] [INSPIRE].

[8] G. Lustermans, W.J. Waalewijn and L. Zeune, Joint transverse momentum and threshold resummation beyond NLL, Phys. Lett. B 762 (2016) 447 [arXiv: 1605.02740] [INSPIRE].

[9] R. Bonciani, S. Catani, M.L. Mangano and P. Nason, Sudakov resummation of multiparton QCD cross-sections, Phys. Lett. B 575 (2003) 268 [hep-ph/0307035] [INSPIRE].

[10] S. Catani, M.L. Mangano and P. Nason, Sudakov resummation for prompt photon production in hadron collisions, JHEP 07 (1998) 024 [hep-ph/9806484] [INSPIRE].

[11] P. Bolzoni, S. Forte and G. Ridolfi, Renormalization group approach to Sudakov resummation in prompt photon production, Nucl. Phys. B 731 (2005) 85 [hep-ph/0504115] [INSPIRE].

[12] S.M. Aybat, L.J. Dixon and G.F. Sterman, The Two-loop anomalous dimension matrix for soft gluon exchange, Phys. Rev. Lett. 97 (2006) 072001 [hep-ph/0606254] [INSPIRE].

[13] E. Gardi and L. Magnea, Factorization constraints for soft anomalous dimensions in QCD scattering amplitudes, JHEP 03 (2009) 079 [arXiv:0901.1091] [INSPIRE].

[14] S. Moch, J.A.M. Vermaseren and A. Vogt, Higher-order corrections in threshold resummation, Nucl. Phys. B 726 (2005) 317 [hep-ph/0506288] [INSPIRE].

[15] C. Muselli, M. Bonvini, S. Forte, S. Marzani and G. Ridolfi, Top Quark Pair Production beyond NNLO, JHEP 08 (2015) 076 [arXiv: 1505.02006] [INSPIRE].

[16] S. Catani, D. de Florian and M. Grazzini, Universality of nonleading logarithmic contributions in transverse momentum distributions, Nucl. Phys. B 596 (2001) 299 [hep-ph/0008184] [INSPIRE]. 
[17] G. Bozzi, S. Catani, D. de Florian and M. Grazzini, Transverse-momentum resummation and the spectrum of the Higgs boson at the LHC, Nucl. Phys. B 737 (2006) 73 [hep-ph/0508068] [INSPIRE].

[18] T. Becher and M. Neubert, Drell-Yan Production at Small $q_{T}$, Transverse Parton Distributions and the Collinear Anomaly, Eur. Phys. J. C 71 (2011) 1665 [arXiv: 1007.4005] [INSPIRE].

[19] S. Catani, L. Cieri, D. de Florian, G. Ferrera and M. Grazzini, Universality of transverse-momentum resummation and hard factors at the NNLO, Nucl. Phys. B 881 (2014) 414 [arXiv:1311.1654] [inSPIRE].

[20] H. Contopanagos, E. Laenen and G.F. Sterman, Sudakov factorization and resummation, Nucl. Phys. B 484 (1997) 303 [hep-ph/9604313] [inSPIRE].

[21] T. Becher, A. Broggio and A. Ferroglia, Introduction to Soft-Collinear Effective Theory, Lect. Notes Phys. 896 (2015) 1 [arXiv:1410.1892] [InSPIRE].

[22] S. Forte and G. Ridolfi, Renormalization group approach to soft gluon resummation, Nucl. Phys. B 650 (2003) 229 [hep-ph/0209154] [INSPIRE].

[23] G. Curci, W. Furmanski and R. Petronzio, Evolution of Parton Densities Beyond Leading Order: The Nonsinglet Case, Nucl. Phys. B 175 (1980) 27 [inSPIRE].

[24] M. Bonvini, S. Forte, G. Ridolfi and L. Rottoli, Resummation prescriptions and ambiguities in SCET vs. direct QCD: Higgs production as a case study, JHEP 01 (2015) 046 [arXiv: 1409.0864] [INSPIRE].

[25] S. Catani and M. Grazzini, Higgs Boson Production at Hadron Colliders: Hard-Collinear Coefficients at the NNLO, Eur. Phys. J. C 72 (2012) 2013 [Erratum ibid. C 72 (2012) 2132] [arXiv:1106.4652] [INSPIRE].

[26] T. Huber and D. Maître, HypExp: A Mathematica package for expanding hypergeometric functions around integer-valued parameters, Comput. Phys. Commun. 175 (2006) 122 [hep-ph/0507094] [INSPIRE].

[27] T. Huber and D. Maître, HypExp 2, Expanding Hypergeometric Functions about Half-Integer Parameters, Comput. Phys. Commun. 178 (2008) 755 [arXiv:0708.2443] [INSPIRE].

[28] S. Albino and R.D. Ball, Soft resummation of quark anomalous dimensions and coefficient functions in MS-bar factorization, Phys. Lett. B 513 (2001) 93 [hep-ph/0011133] [INSPIRE].

[29] S. Catani, D. de Florian and M. Grazzini, Higgs production in hadron collisions: Soft and virtual QCD corrections at NNLO, JHEP 05 (2001) 025 [hep-ph/0102227] [INSPIRE].

[30] T. Becher, M. Neubert and D. Wilhelm, Higgs-Boson Production at Small Transverse Momentum, JHEP 05 (2013) 110 [arXiv:1212.2621] [INSPIRE].

[31] A. Kulesza, G.F. Sterman and W. Vogelsang, Joint resummation for Higgs production, Phys. Rev. D 69 (2004) 014012 [hep-ph/0309264] [INSPIRE].

[32] S. Forte and C. Muselli, High energy resummation of transverse momentum distributions: Higgs in gluon fusion, JHEP 03 (2016) 122 [arXiv:1511.05561] [INSPIRE].

[33] F. Caola, S. Forte, S. Marzani, C. Muselli and G. Vita, The Higgs transverse momentum spectrum with finite quark masses beyond leading order, JHEP 08 (2016) 150 [arXiv: 1606.04100] [INSPIRE]. 
[34] S. Marzani, Combining $Q_{T}$ and small-x resummations, Phys. Rev. D 93 (2016) 054047 [arXiv: 1511.06039] [INSPIRE].

[35] G. Bozzi, S. Catani, G. Ferrera, D. de Florian and M. Grazzini, Transverse-momentum resummation: A perturbative study of $Z$ production at the Tevatron, Nucl. Phys. B 815 (2009) 174 [arXiv:0812.2862] [INSPIRE].

[36] S. Catani, D. de Florian, G. Ferrera and M. Grazzini, Vector boson production at hadron colliders: transverse-momentum resummation and leptonic decay, JHEP 12 (2015) 047 [arXiv: 1507.06937] [INSPIRE]. 